\title{
MODULAR ABELIAN VARIETIES OVER NUMBER FIELDS
}

\author{
XAVIER GUITART AND JORDI QUER
}

\begin{abstract}
The main result of this paper is a characterization of the abelian varieties $B / K$ defined over Galois number fields with the property that the $L$-function $L(B / K ; s)$ is a product of $L$-functions of non-CM newforms over $\mathbb{Q}$ for congruence subgroups of the form $\Gamma_{1}(N)$. The characterization involves the structure of $\operatorname{End}(B)$, isogenies between the Galois conjugates of $B$, and a Galois cohomology class attached to $B / K$.

We call the varieties having this property strongly modular. The last section is devoted to the study of a family of abelian surfaces with quaternionic multiplication. As an illustration of the ways in which the general results of the paper can be applied we prove the strong modularity of some particular abelian surfaces belonging to that family, and we show how to find nontrivial examples of strongly modular varieties by twisting.
\end{abstract}

\section{Introduction}

We will work in the category of abelian varieties up to isogeny, in which the objects are abelian varieties and the morphisms between two varieties $A$ and $B$ are the elements of the $\mathbb{Q}$-vector space $\operatorname{Hom}^{0}(A, B):=\mathbb{Q} \otimes_{\mathbb{Z}} \operatorname{Hom}(A, B)$, where $\operatorname{Hom}(A, B)$ denotes the usual $\mathbb{Z}$-module of homomorphisms between abelian varieties. In particular, isogenies become isomorphisms in our category. We will use the standard Hom, End and Aut to denote morphisms up to isogeny (we will suppress the superscripts to lighten the notation). As usual, a field as an index means morphisms defined over that field. The notation $A \sim B$ will indicate that the abelian varieties $A$ and $B$ are isogenous, and $A \sim_{K} B$ that they are isogenous with an isogeny defined over the field $K$.

Let $f=\sum a_{n} q^{n}$ be a weight-two newform for the congruence subgroup $\Gamma_{1}(N)$, and let $E_{f}=$ $\mathbb{Q}\left(\left\{a_{n}\right\}\right)$ be the number field generated by its Fourier coefficients. Shimura attaches to $f$ an abelian variety $A_{f}$ defined over $\mathbb{Q}$, constructed as a subvariety of the Jacobian $J_{1}(N)$ of the modular curve $X_{1}(N)$. The variety $A_{f}$ has dimension equal to the degree $\left[E_{f}: \mathbb{Q}\right]$, the algebra $\operatorname{End}_{\mathbb{Q}}\left(A_{f}\right)$ of its endomorphisms defined over $\mathbb{Q}$ is isomorphic to the number field $E_{f}$, and the $L$-function $L\left(A_{f} / \mathbb{Q} ; s\right)$ is equivalent (i.e., coincides up to a finite number of Euler factors) with the product $\prod L\left({ }^{\sigma} f ; s\right)$ of the $L$-functions of the Galois conjugates of the form $f$ [21, Section 7.5].

The abelian varieties $A_{f}$ and, more generally, all abelian varieties $A / \mathbb{Q}$ that are isogenous over $\mathbb{Q}$ to $A_{f}$ for some $f$ are known as modular abelian varieties. This modularity property has many important consequences and applications, for example:

- modularity implies the Hasse conjecture for the $L$-function $L(A / \mathbb{Q} ; s)$;

- the theory of Heegner points and results by Gross-Zagier and Kolyvagin produce partial results for the variety $A / \mathbb{Q}$ in the direction of the Birch and Swinnerton-Dyer conjecture;

- the modularity of Frey's elliptic curves can be used to solve certain Diophantine equations of Fermat type.

2010 Mathematics Subject Classification. Primary 11G10, Secondary 11G18, 11F11.

This research was partially supported by grants MTM2009-13060-C02-01 and 2009SGR-1220. 
As a result, modular abelian varieties have been intensively studied and exploited in the last decades. In practice, one can easily compute and work with modular forms and the corresponding modular abelian varieties thanks to the powerful tool provided by the theory of modular symbols: see [3] for elliptic curves and 22] for arbitrary dimension. The computer systems Magma and Sage include packages that are able to perform many explicit computations with those objects.

In the other direction, one would like to characterize the modularity of a given variety $A / \mathbb{Q}$. In the one-dimensional case, the Shimura-Taniyama conjecture predicted that every elliptic curve over $\mathbb{Q}$ is modular, and its proof was completed in [2] by Breuil, Conrad, Diamond and Taylor, using generalizations and variants of the ideas and techniques of Wiles 24 and Taylor-Wiles [23]. In [20] Ribet introduced the concept of a variety of $\mathrm{GL}_{2}$-type as an abelian variety $A / \mathbb{Q}$ for which $\operatorname{End}_{\mathbb{Q}}(A)$ is a number field of degree equal to the dimension of $A$. He generalized ShimuraTaniyama by conjecturing that every variety of $\mathrm{GL}_{2}$-type is modular over $\mathbb{Q}$, and proved that this fact would be a consequence of Serre's conjecture on the modularity of 2-dimensional mod $p$ Galois representations [20, Theorem 4.4]. After the proof of Serre's conjecture by Khare and Wintenberger [11, Theorem 9.1], we now know that modularity of an abelian variety over $\mathbb{Q}$ is equivalent to the property of being of $\mathrm{GL}_{2}$-type.

The abelian varieties of $\mathrm{GL}_{2}$-type are not absolutely simple in general: they factor up to isogeny as products of varieties defined over number fields. After some work done by Elkies in the one-dimensional case and by Ribet in general, in [15] Pyle gave a characterization of the abelian varieties defined over number fields that appear in the absolute decomposition of abelian varieties of $\mathrm{GL}_{2}$-type, which depends on the structure of their endomorphism algebras and on the existence of isogenies between their Galois conjugates. She uses the name building blocks for them (also known as elliptic $\mathbb{Q}$-curves in the one-dimensional case) and generalizes the use of the term modular abelian variety to refer to an abelian variety $B / K$ defined over a number field $K$ that is isogenous to a factor of some $A_{f}$. In this sense, Ribet's generalization of the Shimura-Taniyama conjecture predicts that building blocks and absolutely simple modular abelian varieties are the same things up to isogeny, and now, after the work of Khare and Wintenberger, this is known to be a fact.

In this way one gets a new family of abelian varieties defined over number fields and is tempted to use their modularity in the same way as was done over $\mathbb{Q}$. But the key property of modularity that is used for many of the applications is the relation of the $L$-function of the variety with modular forms, and this property is invariant only by isogeny defined over the base field. Hence, to use modularity in the context of abelian varieties over number fields, it is natural to single out a class of abelian varieties that we will term 'strongly modular.'

Definition 1.1. Let $K$ be a number field. An abelian variety $B / K$ is strongly modular over $K$ if its $L$-function $L(B / K ; s)$ is equivalent to a product of $L$-series of newforms over $\mathbb{Q}$ for $\Gamma_{1}(N)$.

In the next section we give a characterization (Proposition 2.4) of strong modularity as the property that the restriction of scalars to $\mathbb{Q}$ is a product of $\mathrm{GL}_{2}$-type varieties. This can be taken as an alternative definition of strong modularity.

It is important to notice that strong modularity is a much more restrictive concept than that of being just a building block. For example, consider an elliptic curve over the field of rational numbers $B / \mathbb{Q}$. Then, the extension of scalars of $B$ to any number field $K$ is a building block, but $B / K$ is strongly modular only in the case that $K / \mathbb{Q}$ is an abelian extension: in this case we have that $L(B / K ; s)=\prod_{\chi} L(f \otimes \chi ; s)$, where $f$ is the newform such that $L(B / \mathbb{Q} ; s)=L(f ; s)$ and $\chi$ runs over the group of complex characters of $\operatorname{Gal}(K / \mathbb{Q})$. Another less trivial example is the following: consider the elliptic curve $B$ defined over the number field $K=\mathbb{Q}(\sqrt{-3})$ by an 
equation of the type:

$$
Y^{2}=X^{3}+4 a X^{2}+2\left(a^{2}+\sqrt{-3} b\right) X, \quad a, b \in \mathbb{Q} .
$$

Then $B / K$ is a building block but it is not strongly modular; in fact, no curve isogenous to $B$ over $\overline{\mathbb{Q}}$ and defined over the field $K$ can be strongly modular. If we enlarge the base field $K$ to $M=\mathbb{Q}(\sqrt{-3}, \sqrt{-2})$ then the curve $B / M$ obtained by extension of scalars is still not strongly modular but its quadratic twist corresponding to the extension $M(\sqrt{2+\sqrt{6}})$ is strongly modular. This example is obtained in [4, §3] using the results of [16] and [17, and is applied to the study of a family of Diophantine equations of Fermat type. The strong modularity of the twisted model is fundamental for this application. Examples of this same type but in higher dimension are given in the last section of this paper as application of our results.

Strongly modular abelian varieties over number fields can be studied as easily as modular abelian varieties over $\mathbb{Q}$. For instance, their $L$-series satisfy the Hasse conjecture, Heegner cycles and Gross-Zagier type results can be used to compute rational points on them, and they can be used for solving Diophantine equations as in the example cited in the previous paragraph.

Of course, for abelian varieties over number fields there are other concepts of modularity that associate the $L$-function of the variety with more general modular and automorphic forms. The case of abelian varieties over totally real number fields and their relation to Hilbert modular forms is perhaps the best understood situation, and many results generalizing the classical ones are known.

The purpose of the present paper is to understand and characterize the abelian varieties over number fields whose $L$-functions can be obtained in terms of classical elliptic modular forms over $\mathbb{Q}$ for congruence subgroups $\Gamma_{1}(N)$, in the precise sense of our definition of strong modularity. This characterization is given in our main theorem, Theorem 5.3. in terms of the Galois group of the number field and of certain Galois cohomology class attached to the variety. The case of non-CM one-dimensional building blocks, i.e., of $\mathbb{Q}$-curves without complex multiplication, was already studied in [16]. This paper is a generalization of [16] to arbitrary dimension; many ideas and tools we will use here were already introduced in [20], [15] and [16].

It would be desirable to have also methods to produce modular abelian varieties without the use of modular forms, with the modularity being just a consequence of their arithmetic and geometric properties. The one-dimensional case is of course the most well known, and for this reason it has been the main source of applications up to now: all elliptic curves over $\mathbb{Q}$ are strongly modular. Over number fields all CM elliptic curves are modular, and Elkies proved in [5] that non-CM modular elliptic curves are parameterized up to isogeny by the (noncusp, non-CM) rational points of the modular curves $X^{*}(N)$ quotient of $X_{0}(N)$ by the group of Atkin-Lehner involutions, for squarefree values of $N$. A method to explicitly work-out this parametrization when the moduli variety $X^{*}(N)$ is of genus zero or one (this happens for 81 values of $N$ in the range 2-238) is given in [6], and standard conjectures suggest that only a finite number of isogeny classes is missing out of these genus $\leqslant 1$ moduli curves. Once one has a modular elliptic curve the results in [16] can be used to characterize the curves in its isogeny class that are strongly modular, and explicit equations for them may be obtained using the methods developed in [17].

The non-modular construction of higher dimensional strongly modular abelian varieties has been performed up to now only over $\mathbb{Q}$. They appear either as Jacobians of curves $C / \mathbb{Q}$ for which one is able to write down enough endomorphisms defined over $\mathbb{Q}$, or as the varieties corresponding to certain points in moduli varieties, especially Shimura curves. In the last section we consider a family of modular abelian surfaces over number fields that are strictly non-rational examples, in the sense that they cannot be obtained by just extending scalars from varieties defined over 
$\mathbb{Q}$, and we see how the main theorem of this paper can be used to distinguish (and to produce) strongly modular examples.

Remark on complex multiplication. Shimura proved that a variety $A_{f}$ has a factor with complex multiplication if and only if it is isogenous to a power of an elliptic curve with complex multiplication. This is also equivalent to the fact that the newform $f$ admits a twist by a quadratic character whose kernel is the field of complex multiplication of the corresponding elliptic curve. The CM case requires a special treatment and, except for section 2, in which the results hold in complete generality, for the rest of the paper we will tacitly assume that all abelian varieties considered have no CM-factors up to isogeny; when necessary we will stress this condition by saying "non-CM abelian variety."

Acknowledgments. Guitart wants to thank the Max Planck Institute for Mathematics for their hospitality and financial support during his stay at the Institute, where part of the present work has been carried out.

\section{Strong MOdUlarity AND $\mathrm{GL}_{2}$-TYPe}

The purpose of this section is to show that an abelian variety $B / K$ is strongly modular if and only if the abelian variety $A / \mathbb{Q}$ obtained by restriction of scalars $A=\operatorname{Res}_{K / \mathbb{Q}}(B)$ is isogenous over $\mathbb{Q}$ to a product of abelian varieties of $G_{2}$-type. Due to the fact that this last property is the one that plays a key role in this paper, many statements become simpler if we enlarge the definition of $\mathrm{GL}_{2}$-type to include varieties that are not simple over $\mathbb{Q}$.

Definition 2.1. An abelian variety $A / \mathbb{Q}$ is of $\operatorname{GL}_{2}$-type if $\operatorname{End}_{\mathbb{Q}}(A)$ contains a commutative semisimple sub- $\mathbb{Q}$-algebra of $\mathbb{Q}$-dimension equal to $\operatorname{dim} A$.

Note that the standard use of "GL $\mathrm{GL}_{2}$-type" in the literature corresponds to the varieties that satisfy our definition and are simple. The relation between the two concepts is analogous to the relation between arbitrary CM-abelian varieties and the simple ones (cf. [13, p. 29]).

Lemma 2.2. An abelian variety is of $\mathrm{GL}_{2}$-type if and only if all its $\mathbb{Q}$-simple factors are of $\mathrm{GL}_{2}$-type.

Proof. For an abelian variety $A / \mathbb{Q}$ let $A \sim_{\mathbb{Q}} A_{1}^{r_{1}} \times \cdots \times A_{n}^{r_{n}}$ be its decomposition up to $\mathbb{Q}$-isogeny into $\mathbb{Q}$-simple factors. Put $\mathcal{D}_{i}=\operatorname{End}_{\mathbb{Q}}\left(A_{i}\right)$, let $F_{i}$ be the center of $\mathcal{D}_{i}$ and let $t_{i}=\left[\mathcal{D}_{i}: F_{i}\right]^{1 / 2}$ be its index. The decomposition of $\operatorname{End}_{\mathbb{Q}}(A)$ into simple algebras is

$$
\operatorname{End}_{\mathbb{Q}}(A) \simeq \mathrm{M}_{r_{1}}\left(\mathcal{D}_{1}\right) \times \cdots \times \mathrm{M}_{r_{n}}\left(\mathcal{D}_{n}\right),
$$

and the reduced degree of $\operatorname{End}_{\mathbb{Q}}(A)$ over $\mathbb{Q}$ is $\left[\operatorname{End}_{\mathbb{Q}}(A): \mathbb{Q}\right]_{\mathrm{red}}=\sum r_{i} t_{i}\left[F_{i}: \mathbb{Q}\right]$.

If every $A_{i}$ is of $\mathrm{GL}_{2}$-type then $\mathcal{D}_{i}=F_{i}$ has degree $\left[F_{i}: \mathbb{Q}\right]=\operatorname{dim} A_{i}$. Every field extension $E_{i} / F_{i}$ of degree $r_{i}$ can be embedded in the matrix ring $\mathrm{M}_{r_{i}}\left(F_{i}\right)$ and the product $\prod E_{i}$ is a commutative semisimple subalgebra of $\operatorname{End}_{\mathbb{Q}}(A)$ of dimension $\sum\left[E_{i}: \mathbb{Q}\right]=\sum r_{i}\left[F_{i}: \mathbb{Q}\right]=$ $\sum r_{i} \operatorname{dim} A_{i}=\operatorname{dim} A$, hence $A$ is of $\mathrm{GL}_{2}$-type.

For the converse we will make use of the following basic facts about associative algebras: for any semisimple $k$-algebra $\mathcal{A}$ the maximal commutative semisimple subalgebras $E \subseteq \mathcal{A}$ have dimension $\operatorname{dim}_{k} E=[\mathcal{A}: k]_{\text {red }}$, and for every faithful $\mathcal{A}$-module $M$ one has $\operatorname{dim}_{k} M \geqslant[\mathcal{A}: k]_{\text {red }}$ with the equality being possible only if all the simple subalgebras of $\mathcal{A}$ are matrix algebras over fields (cf. [13, Propositions 1.3 and 1.2]). The second fact applied to the space of tangent vectors $\operatorname{Lie}(B / \mathbb{Q})$ of an abelian variety $B / \mathbb{Q}$ gives the inequality $\left[\operatorname{End}_{\mathbb{Q}}(B): \mathbb{Q}\right]_{\mathrm{red}} \leqslant \operatorname{dim} B=$ $\operatorname{dim} \operatorname{Lie}(B / \mathbb{Q})$. 
Assume now that $A$ is of $\mathrm{GL}_{2}$-type. Let $E \subseteq \operatorname{End}_{\mathbb{Q}}(A)$ be a commutative semisimple subalgebra with $[E: \mathbb{Q}]=\operatorname{dim} A$. Then by the previous results

$$
\operatorname{dim} A=[E: \mathbb{Q}] \leqslant\left[\operatorname{End}_{\mathbb{Q}}(A): \mathbb{Q}\right]_{\mathrm{red}} \leqslant \operatorname{dim} A .
$$

Hence each step is an equality and $\operatorname{End}_{\mathbb{Q}}(A)$ is product of matrix algebras over fields; i.e., $t_{i}=1$ for all $i$.

Now, using the inequalities $\left[\operatorname{End}_{\mathbb{Q}}\left(A_{i}\right): \mathbb{Q}\right]_{\mathrm{red}} \leqslant \operatorname{dim} A_{i}$ for every $i$, we have

$$
\operatorname{dim} A=\left[\operatorname{End}_{\mathbb{Q}}(A): \mathbb{Q}\right]_{\mathrm{red}}=\sum r_{i}\left[F_{i}: \mathbb{Q}\right]=\sum r_{i}\left[\operatorname{End}_{\mathbb{Q}}\left(A_{i}\right): \mathbb{Q}\right]_{\mathrm{red}} \leqslant \sum r_{i} \operatorname{dim} A_{i}=\operatorname{dim} A
$$

the equality at each summand follows, from which one deduces $\left[F_{i}: \mathbb{Q}\right]=\operatorname{dim} A_{i}$ for all $i$ and so all simple factors $A_{i}$ are of $\mathrm{GL}_{2}$-type.

Proposition 2.3. An abelian variety $A / \mathbb{Q}$ is strongly modular over $\mathbb{Q}$ if and only if it is of $\mathrm{GL}_{2}$-type.

Proof. If $A / \mathbb{Q}$ is of $\mathrm{GL}_{2}$-type, by the previous lemma we have that $A \sim_{\mathbb{Q}} A_{1}^{r_{1}} \times \cdots \times A_{n}^{r_{n}}$, where the $A_{i}$ 's are $\mathbb{Q}$-simple abelian varieties of $\mathrm{GL}_{2}$-type. Results of Ribet [20, Theorem 4.4] and Khare and Wintenberger [11, Theorem 10.1], together with Faltings's isogeny theorem imply the existence of newforms $f_{i}$ such that $A_{i} \sim_{\mathbb{Q}} A_{f_{i}}$. Then $L(A / \mathbb{Q}, s) \sim \prod L\left(A_{i} / \mathbb{Q}, s\right)^{r_{i}} \sim$ $\prod L\left(A_{f_{i}} / \mathbb{Q}, s\right)^{r_{i}}$, where $\sim$ denotes equivalence of $L$-functions. Since each $L\left(A_{f_{i}} / \mathbb{Q}, s\right)$ is the product of the $L$-functions of the newforms that are Galois conjugates of $f_{i}$, the variety $A$ is strongly modular over $\mathbb{Q}$.

Now we prove the converse. Let $A / \mathbb{Q}$ be a strongly modular abelian variety over $\mathbb{Q}$, and let $f_{1}, \ldots, f_{n}$ be newforms such that $L(A / \mathbb{Q}, s)=\prod L\left(f_{i}, s\right)$. Let $E_{i}$ be the field of Fourier coefficients of $f_{i}$, and denote by $E=E_{1} E_{2} \cdots E_{n}$ the composition. Let $m=[E: \mathbb{Q}]$ and $m_{i}=\left[E: E_{i}\right]$, and denote by $\Sigma_{E}$ and $\Sigma_{E_{i}}$ the corresponding sets of complex embeddings. For every index $i$, the restriction of all the elements of $\Sigma_{E}$ to the field $E_{i}$ gives $m_{i}$ copies of every element of $\Sigma_{E_{i}}$.

We will make use of the following notation: if $S=\sum a_{n} n^{-s}$ is a Dirichlet series with $a_{n} \in$ $\mathbb{C}$ and $\sigma \in \operatorname{Aut}(\mathbb{C})$, we denote by ${ }^{\sigma} S$ the series $\sum{ }^{\sigma} a_{n} n^{-s}$; that is, the series obtained by applying $\sigma$ to the coefficients $a_{n}$. Note that since $L(A / \mathbb{Q}, s)$ has rational coefficients we have that ${ }^{\sigma} L(A / \mathbb{Q}, s)=L(A / \mathbb{Q}, s)$. One has

$$
\begin{aligned}
& L\left(A^{m} / \mathbb{Q}, s\right)=L(A / \mathbb{Q}, s)^{m}=\prod_{\sigma \in \Sigma_{E}}{ }^{\sigma} L(A / \mathbb{Q}, s)=\prod_{\sigma \in \Sigma_{E}} \prod_{i=1}^{n}{ }^{\sigma} L\left(f_{i}, s\right) \\
& \left.=\prod_{i=1}^{n} \prod_{\sigma \in \Sigma_{E}} L{ }^{\sigma} f_{i}, s\right)=\prod_{i=1}^{n} \prod_{\sigma \in \Sigma_{E_{i}}} L\left({ }^{\sigma} f_{i}, s\right)^{m_{i}}=\prod_{i=1}^{n} L\left(A_{f_{i}} / \mathbb{Q}, s\right)^{m_{i}}=L\left(\left(\prod_{i=1}^{n} A_{f_{i}}^{m_{i}}\right) / \mathbb{Q}, s\right) .
\end{aligned}
$$

Then by Faltings's isogeny theorem the varieties $A^{m}$ and $\prod A_{f_{i}}^{m_{i}}$ are isogenous over $\mathbb{Q}$. By the uniqueness of decomposition up to $\mathbb{Q}$-isogeny into the product of $\mathbb{Q}$-simple varieties it follows that $A$ is isogenous over $\mathbb{Q}$ to a product $\prod A_{f_{i}}^{e_{i}}$ for some exponents $e_{i} \geqslant 0$, and thus it is of $\mathrm{GL}_{2}$-type.

For other number fields strong modularity can be reduced to that of the restriction of scalars.

Proposition 2.4. An abelian variety $B / K$ over a number field $K$ is strongly modular over $K$ if and only if $\operatorname{Res}_{K / \mathbb{Q}}(B / K)$ is of $\mathrm{GL}_{2}$-type. 
Proof. The equality of $L$-functions $L(B / K, s)=L\left(\left(\operatorname{Res}_{K / \mathbb{Q}} B\right) / \mathbb{Q}, s\right)$ implies that $B$ is strongly modular over $K$ if and only if $\operatorname{Res}_{K / \mathbb{Q}} B$ is strongly modular over $\mathbb{Q}$, and by the previous proposition this is the case if and only if $\operatorname{Res}_{K / \mathbb{Q}} B$ is of $\mathrm{GL}_{2}$-type.

Combining Lemma 2.2 with this proposition one immediately obtains the following consequence.

Corollary 2.5. An abelian variety is strongly modular over a number field $K$ if and only if all its $K$-simple factors are strongly modular over $K$.

\section{3. $\mathbb{Q}$-ABELIAN VARIETIES}

The absolutely simple factors up to isogeny of non-CM abelian varieties of $\mathrm{GL}_{2}$-type are studied by Ribet in [20] and by Pyle in [15]. A common property of the non-CM abelian varieties of $\mathrm{GL}_{2}$-type and of their simple factors is that the object consisting of the variety together with its endomorphisms has as field of moduli the field of rational numbers. In order to deal with this property the following definitions are useful. For a given abelian variety $B / \overline{\mathbb{Q}}$ and Galois automorphism $\sigma \in G_{\mathbb{Q}}$, an isogeny $\mu_{\sigma}:{ }^{\sigma} B \rightarrow B$ is said to be compatible with the endomorphisms of $B$ if the $\operatorname{map} \operatorname{End}(B) \rightarrow \operatorname{End}(B): \psi \mapsto \mu_{\sigma}{ }^{\circ}{ }^{\sigma} \psi{ }^{\circ} \mu_{\sigma}^{-1}$ is the identity, i.e., if the diagram

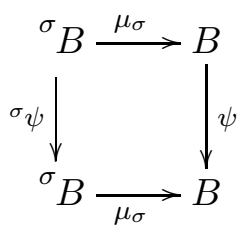

is commutative for every $\psi \in \operatorname{End}(B)$.

Definition 3.1 ([15, p. 194]). A $\mathbb{Q}$-abelian variety (or just $\mathbb{Q}$-variety for short) is an abelian variety $B / \overline{\mathbb{Q}}$ such that for every $\sigma \in G_{\mathbb{Q}}$ there exists an isogeny $\mu_{\sigma}$ : ${ }^{\sigma} B \rightarrow B$ compatible with $\operatorname{End}(B)$.

Let $F$ be the center of the endomorphism algebra $\operatorname{End}(B)$. It is easily seen that if the isogeny $\mu_{\sigma}:{ }^{\sigma} B \rightarrow B$ is compatible with $\operatorname{End}(B)$, then all isogenies between these two varieties compatible with $\operatorname{End}(B)$ are the maps of the form $\psi \circ \mu_{\sigma}$ for $\psi \in F^{*}$; also, if $B$ is a $\mathbb{Q}$-abelian variety, then all its endomorphisms belonging to the center $F$ are defined over every field of definition for $B$.

The cocycle class $\left[c_{B}\right]$. Let $B / \overline{\mathbb{Q}}$ be a $\mathbb{Q}$-variety. Since the variety is defined over some number field one can always choose a set of isogenies $\left\{\mu_{\sigma}:{ }^{\sigma} B \rightarrow B\right\}_{\sigma \in G_{\mathbb{Q}}}$ compatible with the endomorphisms of $B$ that is locally constant. Let $c_{B}$ be the map defined as

$$
c_{B}: G_{\mathbb{Q}} \times G_{\mathbb{Q}} \rightarrow F^{*}, \quad c_{B}(\sigma, \tau)=\mu_{\sigma^{\circ}}{ }^{\sigma} \mu_{\tau} \circ \mu_{\sigma \tau}^{-1} .
$$

In the following lemma we state some of the properties of $c_{B}$, which can be straightforwardly checked.

Lemma 3.2. The map $c_{B}$ is a well-defined continuous 2 -cocycle on $G_{\mathbb{Q}}$ with values in the group $F^{*}$, considered as a $G_{\mathbb{Q}}$-module with trivial action, and the cohomology class $\left[c_{B}\right] \in H^{2}\left(G_{\mathbb{Q}}, F^{*}\right)$ does not depend on the locally constant set of isogenies used to define the cocycle $c_{B}$. Moreover, the class $\left[c_{B}\right]$ depends only on the $\overline{\mathbb{Q}}$-isogeny class of $B$. 
This invariant $\left[c_{B}\right]$ gives the obstruction to descend up to isogeny the variety and its endomorphisms over a given number field. The following result is stated as Proposition 5.2 in [15] for $\mathbb{Q}$-abelian varieties that are building blocks, but in the proof given there the structure of the endomorphism algebra plays no role, so we state it here in full generality.

Proposition 3.3 (Ribet-Pyle). Let $B / \overline{\mathbb{Q}}$ be a $\mathbb{Q}$-variety and let $K$ be a number field. There exists an abelian variety defined over $K$ and with all the endomorphisms defined over $K$ that is isogenous to $B$ if and only if $\left[c_{B}\right]$ belongs to the kernel of the restriction map $\operatorname{Res}: H^{2}\left(G_{\mathbb{Q}}, F^{*}\right) \rightarrow$ $H^{2}\left(G_{K}, F^{*}\right)$.

Generalizing the terminology introduced in [16] for elliptic $\mathbb{Q}$-curves we will say that a $\mathbb{Q}$ variety $B$ is completely defined over a Galois number field $K$ if the variety $B$ and all its endomorphisms are defined over $K$, and there exist isogenies between Galois conjugates compatible with $\operatorname{End}(B)$ that are all of them defined over $K$. In this case, let $\mu_{s}:{ }^{s} B \rightarrow B$ be an isogeny compatible with the endomorphisms of $B$ for each element $s \in G=\operatorname{Gal}(K / \mathbb{Q})$. Then, as for (3.1), one sees that the map

$$
c_{B / K}: G \times G \rightarrow F^{*}, \quad c_{B / K}(s, t)=\mu_{s} \circ{ }^{s} \mu_{t} \circ \mu_{s t}^{-1}
$$

is a well-defined 2-cocycle on $G$ with values in the trivial $G$-module $F^{*}$ whose cohomology class $\left[c_{B / K}\right] \in H^{2}\left(K / \mathbb{Q}, F^{*}\right)$ is an invariant of the $K$-isogeny class of the variety $B$.

Proposition 3.4. Let $B / \overline{\mathbb{Q}}$ be a $\mathbb{Q}$-variety and let $K$ be a Galois number field. There exists an abelian variety completely defined over $K$ that is isogenous to $B$ if and only if $\left[c_{B}\right]$ belongs to the image of the inflation map Inf: $H^{2}\left(K / \mathbb{Q}, F^{*}\right) \rightarrow H^{2}\left(G_{\mathbb{Q}}, F^{*}\right)$.

Moreover, if $\left[c_{B}\right]=\operatorname{Inf}([c])$ for some cocycle class $[c] \in H^{2}\left(K / \mathbb{Q}, F^{*}\right)$, then there exists such a variety $B_{0} / K$ such that $\left[c_{B_{0} / K}\right]=[c]$.

Proof. Since the image of the inflation lies in the kernel of the restriction, by Proposition 3.3 we can suppose that $B$ and all of its endomorphisms are defined over $K$.

Assume that $\left[c_{B}\right]=\operatorname{Inf}([c])$. Modifying the 2-cocycle $c$ by a coboundary we can assume that it is normalized, i.e., takes the value $c(1,1)=1$, and as a consequence of the cocycle condition this implies that also $c(s, 1)=c(1, s)=1$ for every $s \in \operatorname{Gal}(K / \mathbb{Q})$. Moreover, by changing the choice of isogenies compatible with $\operatorname{End}(B)$ used in (3.1) to define $c_{B}$, we can also suppose that $\inf (c)$ coincides with the cocycle $c_{B}$. This implies that $c_{B}(\sigma, \tau)=1$ whenever $\sigma$ or $\tau$ belong to the subgroup $G_{K}$. It follows that the map $\sigma \mapsto \mu_{\sigma}$ is a one-cocycle on the group $G_{K}$ with values in the group $\operatorname{Aut}(B)$, viewed as a module with the natural Galois action of $G_{K}$, which is in fact the trivial action since all the elements of $\operatorname{End}(B)$ are defined over $K$.

Let $B_{0}$ be the twist of $B$ by this one-cocycle: it is an abelian variety $B_{0}$ defined over $K$ together with an isogeny $\kappa: B \rightarrow B_{0}$ such that $\mu_{\sigma}=\kappa^{-1}{ }_{\circ} \sigma \kappa$ for all $\sigma \in G_{K}$. We will see that this variety satisfies the conditions of the proposition.

Every endomorphism of $B_{0}$ is of the form $\kappa^{\circ} \psi \circ \kappa^{-1}$ for some $\psi \in \operatorname{End}(B)$. Since all endomorphisms of $B$ are defined over $K$ and the isogenies $\mu_{\sigma}$ are compatible with $\operatorname{End}(B)$, for every $\sigma \in G_{K}$ one has

$$
{ }^{\sigma}\left(\kappa \circ \psi \circ \kappa^{-1}\right)={ }^{\sigma} \kappa \circ{ }^{\sigma} \psi{ }^{\circ}{ }^{\sigma} \kappa^{-1}=\kappa \circ \mu_{\sigma}{ }^{\sigma}{ }^{\sigma} \psi \circ \mu_{\sigma}^{-1} \circ \kappa^{-1}=\kappa \circ \psi \circ \kappa^{-1}
$$

and the endomorphisms of $B_{0}$ are defined over $K$ too.

A calculation shows that the maps $\nu_{\sigma}:=\kappa^{\circ} \mu_{\sigma}{ }^{\circ}{ }^{\sigma} \kappa^{-1}$ are isogenies ${ }^{\sigma} B_{0} \rightarrow B_{0}$ compatible with $\operatorname{End}\left(B_{0}\right)$ for every $\sigma \in G_{\mathbb{Q}}$, and the relation of $\mu_{\sigma}=\kappa^{-1}{ }^{\sigma} \kappa$ for elements $\sigma \in G_{K}$ shows that $\nu_{\sigma}=1$ for the $\sigma$ fixing the field $K$. The cocycle $c_{B_{0}}$ computed from this set of isogenies is related to $c_{B}$ by $c_{B_{0}}(\sigma, \tau)=\kappa^{\circ} c_{B}(\sigma, \tau) \circ \kappa^{-1}$ for all $\sigma, \tau \in G_{\mathbb{Q}}$. Since $c_{B}$ is the inflation of $c$ and 
this cocycle is normalized, one deduces that $c_{B_{0}}(\sigma, \tau)=1$ if either $\sigma$ or $\tau$ belong to the subgroup $G_{K}$. Applying this fact to a pair $\sigma \in G_{\mathbb{Q}}$ and $\tau \in G_{K}$ one deduces that

$$
c_{B_{0}}(\sigma, \tau)=\nu_{\sigma}{ }^{\circ} \nu_{\tau} \circ \nu_{\sigma \tau}^{-1}=\nu_{\sigma}{ }^{\circ} \nu_{\sigma \tau}^{-1}=1 \Rightarrow \nu_{\sigma \tau}=\nu_{\sigma}
$$

which means that $\nu_{\sigma}$ depends only on the action of $\sigma$ on $K$ (i.e., on the class of $\sigma$ modulo the normal subgroup $G_{K}$ ). Now, applying the identity to a pair $\sigma \in G_{K}$ and $\tau \in G_{\mathbb{Q}}$ one has

$$
\nu_{\sigma}{ }^{\circ} \nu_{\tau} \circ \nu_{\sigma \tau}^{-1}={ }^{\sigma} \nu_{\tau} \circ \nu_{\sigma \tau}^{-1}={ }^{\sigma} \nu_{\tau} \circ \nu_{\tau}^{-1}=1 \Rightarrow{ }^{\sigma} \nu_{\tau}=\nu_{\tau}
$$

proving that the isogenies $\nu_{\sigma}$ are also defined over $K$ for every $\sigma \in G_{\mathbb{Q}}$.

Finally, for every element $s \in \operatorname{Gal}(K / \mathbb{Q})$ let $\nu_{s}$ be the isogeny $\nu_{\sigma}$ for any $\sigma \in G_{\mathbb{Q}}$ whose action on $K$ is given by the element $s$. In this way one obtains a set of isogenies compatible with $\operatorname{End}\left(B_{0}\right)$ defined over the field $K$ and the cocycle $c_{B_{0} / K}$ computed using this set is the cocycle $c_{B_{0} / K}(s, t)=\kappa \circ c(s, t) \circ \kappa^{-1}$. Hence, under the isomorphism between the centers of the endomorphisms of the varieties $B_{0}$ and $B$ given by conjugation by the isogeny $\kappa$ between them, the cohomology class $\left[c_{B_{0} / K}\right]$ is the class $[c]$ we started with.

Simple $\mathbb{Q}$-varieties of the first kind. Up to now we have put no restrictions in the $\mathbb{Q}$ abelian varieties considered; in particular we have not assumed the varieties to be simple. If $B \sim \prod B_{i}^{m_{i}}$ is the decomposition up to isogeny into simple varieties then it is easy to see that $B$ is a $\mathbb{Q}$-abelian variety if and only if all its simple factors have this property, but we will not need this fact here. For the study of the modular abelian varieties we are interested in, the case of interest is when the center $F$ of $\operatorname{End}(B)$ is a totally real number field; this is equivalent to say that the previous decomposition has a unique simple factor that is a variety of the first kind in the standard terminology employed for the classification of simple abelian varieties according to the type of their endomorphism algebras as algebras with involution (cf. [14, p. 193]). We also recall that the endomorphism algebras of simple varieties of the first kind are either a totally real number field (type I varieties) or a quaternion algebra over such field, that may be either totally indefinite (type II) or totally definite (type III). So we assume from now on that $F=Z(\operatorname{End}(B))$ is a totally real number field; we could also assume that $B$ is absolutely simple but in fact that is not necessary and everything below works for varieties that are powers of simple varieties of the first kind.

Generalizing to our situation the definitions given first by Ribet in [19, p. 113] for simple varieties of type I, and then by Pyle in [15, p. 218] for building blocks, one can define for every isogeny $\mu_{\sigma}:{ }^{\sigma} B \rightarrow B$ compatible with $\operatorname{End}(B)$ its "degree" $\delta\left(\mu_{\sigma}\right)$, which is a totally positive element of the field $F$ whose reduced norm as an element of the $\mathbb{Q}$-algebra $\operatorname{End}(B)$ is the usual degree of the isogeny $\mu_{\sigma}$. Moreover, from the definition of this map and the fact that the Rosati involution fixes the elements of the center $F$ one gets, exactly as in [19, 15], the following identity:

$$
c_{B}(\sigma, \tau)^{2}=\delta\left(\mu_{\sigma}\right) \delta\left(\mu_{\tau}\right) \delta\left(\mu_{\sigma \tau}\right)^{-1},
$$

showing that the cohomology class $\left[c_{B}\right]$ belongs to the 2-torsion subgroup $H^{2}\left(G_{\mathbb{Q}}, F^{*}\right)[2]$.

Now, the structure of the group $H^{2}\left(G_{\mathbb{Q}}, F^{*}\right)[2]$ is particularly simple and a number of consequences about fields of definition can be deduced just by looking at it. As it is described in [19, p. 114] (see also [18, Section 2]), if one starts with any group isomorphism $F^{*} \simeq\{ \pm 1\} \times F^{*} /\{ \pm 1\}$, and using basic facts of group cohomology, one obtains a decomposition

$$
H^{2}\left(G_{\mathbb{Q}}, F^{*}\right)[2] \simeq H^{2}\left(G_{\mathbb{Q}},\{ \pm 1\}\right) \times \operatorname{Hom}\left(G_{\mathbb{Q}}, F^{*} /\{ \pm 1\} F^{* 2}\right)
$$

under which every 2-torsion cohomology class $\xi \in H^{2}\left(G_{\mathbb{Q}}, F^{*}\right)$ has two components $\xi=\left(\xi_{ \pm}, \bar{\xi}\right)$. The sign component $\xi_{ \pm} \in H^{2}\left(G_{\mathbb{Q}},\{ \pm 1\}\right) \simeq \mathrm{Br}_{2}(\mathbb{Q})$ is an element of the 2-torsion of the Brauer group of $\mathbb{Q}$. The degree component $\bar{\xi}$ is a group homomorphism $G_{\mathbb{Q}} \rightarrow F^{*} /\{ \pm 1\} F^{* 2}$. Note 
that the decomposition of the cohomology group depends on the decomposition of the (trivial) $G_{\mathbb{Q}^{-}}$-module $F^{*}$ we have chosen, but it is easy to see that the degree component does not depend on it, and also that, for the classes $\left[c_{B}\right]$ attached to $\mathbb{Q}$-varieties $B$, the degree component is just the map $\sigma \mapsto \delta\left(\mu_{\sigma}\right) \bmod \{ \pm 1\} F^{* 2}$ (hence the name).

Given an element $\xi \in H^{2}\left(G_{\mathbb{Q}}, F^{*}\right)[2]$ we will denote by $K_{P}$ the field fixed by the kernel of the degree component $\bar{\xi}$; since this morphism takes values in a 2-torsion group, the field $K_{P}$ is an abelian extension of exponent 2 of the field $\mathbb{Q}$.

Proposition 3.5. Let $B$ be a $\mathbb{Q}$-abelian variety with $F=Z(\operatorname{End}(B))$ a totally real number field. Let $K_{P}$ be the field fixed by the kernel of $\overline{\left[c_{B}\right]}$.

(1) If $B_{0} \sim B$ with $B_{0}$ and $\operatorname{End}\left(B_{0}\right)$ defined over $K$ then $K_{P} \subseteq K$.

(2) There exist isogenous varieties $B_{0} \sim B$ defined over fields of the form $K=K_{P} \cdot \mathbb{Q}(\sqrt{a})$ for some a $\in \mathbb{Q}$, with $\operatorname{End}\left(B_{0}\right)$ also defined over $K$.

(3) There exist isogenous varieties $B_{0} \sim B$ completely defined over fields of the form $K=$ $K_{P} \cdot \mathbb{Q}(\sqrt{a}, \sqrt{b})$ for some $a, b \in \mathbb{Q}$.

Proof. The decomposition (3.4) has analogues for the group $H^{2}\left(G_{K}, F^{*}\right)$ [2] for every number field $K$, and for the group $H^{2}\left(K / \mathbb{Q}, F^{*}\right)[2]$ for every Galois number field $K$. The restriction and inflation maps respect the corresponding decompositions. It follows that the class $\left[c_{B}\right]$ belongs to the kernel (resp. the image) of the restriction to the first group (resp. of the inflation from the second group) if and only if the two components sign and degree belong to the corresponding kernels (resp. images).

As for the degree component $\overline{\left[c_{B}\right]} \in \operatorname{Hom}\left(G_{\mathbb{Q}}, F^{*} /\{ \pm 1\} F^{* 2}\right)$ each of the two conditions either on the inflation or on the restriction are equivalent to the fact that $K_{P} \subseteq K$. Every element of $H^{2}\left(G_{\mathbb{Q}},\{ \pm 1\}\right) \simeq \mathrm{Br}_{2}(\mathbb{Q})$ can be identified with a quaternion algebra, that can be written as a pair $(a, b)_{\mathbb{Q}}$ with $a, b \in \mathbb{Q}^{*}$, using the standard notation. Such an element always can be trivialized by restriction to a (at most) quadratic extension, for example the extension $\mathbb{Q}(\sqrt{a})$. Also, this element can be inflated from a cohomology class defined on the Galois group of a (at most) biquadratic extension, for example the extension $\mathbb{Q}(\sqrt{a}, \sqrt{b})$ (see [17, Section 2]).

In [19] Ribet proved that for varieties with $\operatorname{End}(B)=F$ a totally real number field of odd degree $[F: \mathbb{Q}]=\operatorname{dim} B$ the field $K_{P}$ already trivializes the sign component, so that there are always $B_{0} \sim B$ with $\operatorname{End}\left(B_{0}\right)$ defined over $K_{P}$. It is easily seen that his argument works in fact for all $\mathbb{Q}$-varieties of the first kind of odd dimension without the assumption on the size of their endomorphisms. On the contrary, for even dimension this fact is not true any more, as the modular examples given in [18] show.

\section{4. $K$-BUILDING BLOCKS}

Since we want to study abelian varieties over a number field $K$ that are quotients up to $K$ isogeny of varieties of $\mathrm{GL}_{2}$-type, we slightly adapt the definition of "building block" given by Pyle in [15, p. 195], in order to keep track of their decomposition over $K$ and not merely over $\overline{\mathbb{Q}}$.

Definition 4.1. Let $K / \mathbb{Q}$ be a Galois extension. We say that a (non-CM) abelian variety $B / K$ is a $K$-building block if

(1) $B$ is a $\mathbb{Q}$-variety admitting isogenies $\mu_{\sigma}:{ }^{\sigma} B \rightarrow B$ compatible with $\operatorname{End}(B)$ defined over $K$ for every $\sigma \in G_{\mathbb{Q}}$, and

(2) $\operatorname{End}_{K}(B)$ is a division algebra with center a number field $E$, having index $t \leqslant 2$ and reduced degree $t[E: \mathbb{Q}]=\operatorname{dim} B$. 
We note the following remarks:

- The requirement that $\operatorname{End}_{K}(B)$ is a division algebra implies that $K$-building blocks are $K$-simple abelian varieties, but they may factor over larger fields.

- The $\mathbb{Q}$-building blocks are the (non-CM) $\mathbb{Q}$-simple abelian varieties of $\mathrm{GL}_{2}$-type.

- The $\overline{\mathbb{Q}}$-building blocks are the building blocks in the sense of Pyle's definition; we will also use this terminology without a prefix field sometimes.

- For $\overline{\mathbb{Q}}$-building blocks the field $E$ is necessarily a totally real field, equal to the center of $\operatorname{End}(B)$. In general it may be either totally real or a CM-field, and the center $F$ of $\operatorname{End}(B)$ is a (necessarily totally real) subfield of $E$.

- We do not require $B$ to have all its endomorphisms defined over $K$. This means that a $K$-building block is not necessarily a $\mathbb{Q}$-variety completely defined over $K$.

Proposition 4.2. Let $A / \mathbb{Q}$ be a $\mathbb{Q}$-simple abelian variety of $\mathrm{GL}_{2}$-type without $C M$ and let $K / \mathbb{Q}$ be a Galois extension. Then the extension of scalars $A / K$ is $K$-isogenous to a power $B^{n}$ of a $K$-building block $B / K$.

Proof. Let $A \sim_{K} B_{1}^{n_{1}} \times \cdots \times B_{r}^{n_{r}}$ be the decomposition up to $K$-isogeny into $K$-simple varieties. Since $\operatorname{End}_{\mathbb{Q}}(A)$ is a subfield of $\operatorname{End}(A)$ it acts on each isotypical factor $B_{i}^{n_{i}}$, hence $\left[\operatorname{End}_{\mathbb{Q}}(A)\right.$ : $\mathbb{Q}] \mid 2 n_{i} \operatorname{dim} B_{i} . \quad$ But $\left[\operatorname{End}_{\mathbb{Q}}(A): \mathbb{Q}\right]=\operatorname{dim} A=\sum n_{i} \operatorname{dim} B_{i}$, and this implies that either $\left[\operatorname{End}_{\mathbb{Q}}(A): \mathbb{Q}\right]=n_{i} \operatorname{dim} B_{i}$ or $\left[\operatorname{End}_{\mathbb{Q}}(A): \mathbb{Q}\right]=2 n_{i} \operatorname{dim} B_{i}$ for each index. The second case is not possible since we are assuming that no subvariety of $A$ has $\mathrm{CM}$, and so $\left[\operatorname{End}_{\mathbb{Q}}(A): \mathbb{Q}\right]=$ $n_{i} \operatorname{dim} B_{i}$, which implies that there is only one isotypical factor and $A \sim_{K} B^{n}$.

Next, we prove that $\operatorname{End}_{\mathbb{Q}}(A)$ is a maximal subfield of $\operatorname{End}_{K}(A)$ or, equivalently, that $\operatorname{End}_{\mathbb{Q}}(A)$ is its own centralizer in $\operatorname{End}_{K}(A)$. Let $\varphi$ be an element of $\operatorname{End}_{K}(A)$ that commutes with $\operatorname{End}_{\mathbb{Q}}(A)$. The image $\varphi(A)$ is isogenous to $B^{r}$ for some $r$. Since $\varphi$ commutes with $\operatorname{End}_{\mathbb{Q}}(A)$, the field $\operatorname{End}_{\mathbb{Q}}(A)$ acts on $B^{r}$, and this implies that $\left[\operatorname{End}_{\mathbb{Q}}(A): \mathbb{Q}\right] \mid 2 r \operatorname{dim} B$. This gives only two options: either $\left[\operatorname{End}_{\mathbb{Q}}(A): \mathbb{Q}\right]=r \operatorname{dim} B$ or $\left[\operatorname{End}_{\mathbb{Q}}(A): \mathbb{Q}\right]=2 r \operatorname{dim} B$. Again, the second is not allowed since $B^{r}$ can not have CM. This means that $r=n$ and $\varphi$ is an isogeny. Hence, $C\left(\operatorname{End}_{\mathbb{Q}}(A)\right)$ is a field and therefore $C\left(\operatorname{End}_{\mathbb{Q}}(A)\right)=\operatorname{End}_{\mathbb{Q}}(A)$.

Set $E=Z\left(\operatorname{End}_{K}(B)\right)$ and let $t$ be the index of $\operatorname{End}_{K}(B)$. We can prove now that $t[E: \mathbb{Q}]=$ $\operatorname{dim} B$. This comes from the decomposition $A \sim_{K} B^{n}$, which translates into an isomorphism $\operatorname{End}_{K}(A) \simeq \mathrm{M}_{n}\left(\operatorname{End}_{K}(B)\right)$. Since $\operatorname{End}_{\mathbb{Q}}(A)$ is a maximal subfield of $\operatorname{End}_{K}(A)$ taking dimensions over $E$ we have that $\left[\operatorname{End}_{\mathbb{Q}}(A): E\right]=n t$, and multiplying both sides of this expression by $[E: \mathbb{Q}]$ it gives $\left[\operatorname{End}_{\mathbb{Q}}(A): \mathbb{Q}\right]=n t[E: \mathbb{Q}]$. Since $\left[\operatorname{End}_{\mathbb{Q}}(A): \mathbb{Q}\right]=\operatorname{dim} A=n \operatorname{dim} B$ we see that $\operatorname{dim} B=t[E: \mathbb{Q}]$.

Since $B$ is $K$-simple, $\operatorname{End}_{K}(B)$ is a division algebra acting on $H_{1}(B, \mathbb{Q})$; therefore $\left[\operatorname{End}_{K}(B)\right.$ : $\mathbb{Q}] \mid 2 \operatorname{dim} B$. This means that $t^{2}[E: \mathbb{Q}] \mid 2 \operatorname{dim} B$, and by the relation $t[E: \mathbb{Q}]=\operatorname{dim} B$ we see that $t \operatorname{dim} B \mid 2 \operatorname{dim} B$, showing that $t \leqslant 2$.

Finally, we have to show that $B$ is a $\mathbb{Q}$-variety with isogenies between Galois conjugates defined over $K$. The argument is the same as in the proof of Proposition 1.4 in [15], but starting from an isogeny between $A_{K}$ and $B^{n}$ defined over $K$. It only has to be noticed that the isogenies $\alpha(\sigma): A \rightarrow A$ that appear in that proof are defined over $\mathbb{Q}$, and this implies that the isogenies defined on page 195 of [15] are defined over $K$.

Corollary 4.3. If a $K$-simple variety is strongly modular over a Galois number field $K$, then it is a K-building block.

Proof. Let $B$ be a $K$-simple strongly modular variety. By Proposition $[2.3$, since $B$ is strongly modular over $K, \operatorname{Res}_{K / \mathbb{Q}}(B)$ is of $\mathrm{GL}_{2}$-type. Since $\operatorname{Res}_{K / \mathbb{Q}}(B) \sim_{K} \prod_{s \in \operatorname{Gal}(K / \mathbb{Q})}{ }^{s} B$, the variety 
$B$ is a $K$-simple factor of a $\mathbb{Q}$-simple variety of $\mathrm{GL}_{2}$-type, and then it is a $K$-building block by the previous proposition.

The converse of this corollary is not true: a $K$-building block needs extra conditions to be strongly modular. This conditions are related to a cohomology class $\left[c_{B / K}\right]$ attached to $B$ that is defined in a similar way than (3.2) as follows. Let $B$ be a $K$-building block over a Galois number field $K$; put $G=\operatorname{Gal}(K / \mathbb{Q}), E=Z\left(\operatorname{End}_{K}(B)\right)$ and $F=Z(\operatorname{End}(B))$. Let $\left\{\mu_{\sigma}\right\}_{\sigma \in G_{\mathbb{Q}}}$ be a set of isogenies compatible with $\operatorname{End}(B)$ defined over $K$. For each $s \in G$ choose a representative $\tilde{s}$ in $G_{\mathbb{Q}}$, and define

$$
c_{B / K}: G \times G \rightarrow E^{*}, \quad c_{B / K}(s, t)=\mu_{\tilde{s}^{\circ}}{ }^{\tilde{s}} \mu_{\tilde{t}^{\circ}} \mu_{\tilde{s t}}^{-1} .
$$

When all the endomorphisms of $B$ are defined over $K$ this cocycle $c_{B / K}(s, t)$ coincides with (3.2). Since now we are not requiring the field $K$ to be a field of definition of all the endomorphisms of $B$, we can only guarantee that $c_{B / K}(s, t)$ lies in $E^{*}$ but not in $F^{*}$ as it happens when the variety is completely defined over $K$. In the next lemma we state the main properties of this cocycle.

Lemma 4.4. The map $c_{B / K}$ is a 2-cocycle on $G$ with values in $E^{*}$, considered as a module with trivial action. The cohomology class $\left[c_{B / K}\right] \in H^{2}\left(K / \mathbb{Q}, E^{*}\right)$ depends neither on the lift $s \mapsto \tilde{s}$ nor on the choice of the isogenies $\mu_{\tilde{s}}$. Moreover, the inflation of $\left[c_{B / K}\right]$ to $H^{2}\left(G_{\mathbb{Q}}, E^{*}\right)$ coincides with the image of $\left[c_{B}\right]$ under the morphism $H^{2}\left(G_{\mathbb{Q}}, F^{*}\right) \rightarrow H^{2}\left(G_{\mathbb{Q}}, E^{*}\right)$ induced by the embedding $F^{*} \hookrightarrow E^{*}$.

Proof. Let $\varphi$ be an element of $\operatorname{End}_{K}(B)$. Since $\tilde{s t}=\tilde{s} \tilde{t} \tau$ for some $\tau \in G_{K}$ we have

$$
\begin{aligned}
& c_{B / K}(s, t) \circ \varphi=\mu_{\tilde{s}}{ }^{\tilde{s}} \mu_{\tilde{t}} \mu_{\tilde{s t}}^{-1} \circ \varphi=\mu_{\tilde{s}}{ }^{\tilde{s}} \mu_{\tilde{t}}{ }^{\widetilde{s} t} \varphi \circ \mu_{\tilde{s t}}^{-1}=
\end{aligned}
$$

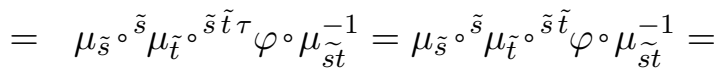

$$
\begin{aligned}
& =\mu_{\tilde{s}}{ }^{\circ}{ }^{\tilde{s}}{ }^{\circ}{ }^{\tilde{s}} \mu_{\tilde{t}}{ }^{\circ} \mu_{\tilde{s} t}^{-1}=\varphi \circ \mu_{\tilde{s}}{ }^{\circ} \mu_{\tilde{t}^{\circ}} \mu_{\tilde{s} t}^{-1}=\varphi \circ c_{B / K}(s, t) \text {, }
\end{aligned}
$$

and this shows that $c_{B}(s, t)$ lies in $E$. In the same way we can prove the cocycle condition, and the independence on the set $\left\{\mu_{\sigma}\right\}_{\sigma \in G_{\mathbb{Q}}}$ is seen in an analogous way than for the case of the cocycle $c_{B}$.

Observe that, if $\sigma \in G_{\mathbb{Q}}$ is such that $\left.\sigma\right|_{K}=\left.\tilde{s}\right|_{K}$ then $\mu_{\sigma}{ }^{\circ} \mu_{\tilde{s}}^{-1}$ commutes with the elements in $\operatorname{End}_{K}(B)$; therefore, we can write $\mu_{\sigma}=\lambda_{\sigma}{ }^{\circ} \mu_{\tilde{s}}$ for some $\lambda_{\sigma} \in E^{*}$. Using this it is immediate to see that the use of another lift from $\operatorname{Gal}(K / \mathbb{Q})$ to $G_{\mathbb{Q}}$ would modify the cocycle $c_{B / K}$ by a coboundary.

It remains to prove the last statement in the lemma. Take $\sigma, \tau \in G_{\mathbb{Q}}$ and put $s=\left.\sigma\right|_{K}$, $t=\left.\tau\right|_{K}$. We use the same name for the cocycles and for their images for the morphisms involved; namely, $c_{B / K}$ is the inflation to $G_{\mathbb{Q}}$ of $c_{B / K}$ and $c_{B}$ is the image of $c_{B}$ in $Z^{2}\left(G_{\mathbb{Q}}, E^{*}\right)$. By the definitions $c_{B}(\sigma, \tau)=\mu_{\sigma}{ }^{\sigma} \sigma \mu_{\tau} \circ \mu_{\sigma \tau}^{-1}$ and $c_{B / K}(\sigma, \tau)=\mu_{\tilde{s}^{\circ}} \tilde{s}^{\circ} \mu_{\tilde{t}^{\circ}} \mu_{\tilde{s} t}^{-1}$. Since $\sigma_{\mid K}=\tilde{s}_{\mid K}$ we see that $\mu_{\sigma}=\mu_{\tilde{s}} \circ \lambda_{\sigma}$ for some $\lambda_{\sigma} \in E$. Now $c_{B}(\sigma, \tau)=c_{B / K}(\sigma, \tau) \circ \lambda_{\sigma} \circ \lambda_{\tau} \circ \lambda_{\sigma \tau}^{-1}$ and the two cocycles are cohomologous.

Restriction of scalars of $K$-building blocks. Our objective now is to compute the endomorphism algebra $\operatorname{End}_{\mathbb{Q}}\left(\operatorname{Res}_{K / \mathbb{Q}}(B)\right)$ for a $K$-building block $B$. What we obtain is a generalization of the expression found by Ribet in [20, Lemma 6.4] for the case of $\mathbb{Q}$-curves, giving the algebra as a twisted group algebra. The main difference is that in our case the algebra is obtained by a construction that mimics the standard twisted group algebra definition, which we first describe in abstract terms. 
Let $\mathcal{A}$ be a central $E$-algebra and let $c \in Z^{2}\left(G, E^{*}\right)$ be a two-cocycle on a finite group $G$ with values in the multiplicative group $E^{*}$ viewed as a module with trivial action. One defines the $E$-algebra $\mathcal{A}^{c}[G]$ by generalizing the usual definition of twisted group algebra: it is the free left $\mathcal{A}$-module $\oplus_{s \in G} \mathcal{A} \cdot \lambda_{s}$ with basis a set of symbols $\lambda_{s}$ indexed by the elements $s \in G$ and multiplication defined by the relations:

$$
\begin{aligned}
a \cdot \lambda_{s} & =\lambda_{s} \cdot a, \quad \text { for } \quad a \in \mathcal{A}, \\
\lambda_{s} \cdot \lambda_{t} & =c(s, t) \cdot \lambda_{s t} .
\end{aligned}
$$

The cocycle condition for $c$ is used to check that this definition makes sense and produces an associative algebra, and of course its isomorphism class does depend only on the cohomology class of the cocycle $c$. This algebra is related with the twisted group algebra $E^{c}[G]$ through the following isomorphism:

$$
\mathcal{A}^{c}[G] \simeq \mathcal{A} \otimes_{E} E^{c}[G] \quad \text { as } \quad E \text {-algebras. }
$$

Indeed, if we let $E^{c}[G]=\oplus_{s \in G} E \cdot \lambda_{s}$ then the map $a \otimes \sum x_{s} \cdot \lambda_{s} \mapsto \sum\left(a x_{s}\right) \cdot \lambda_{s}: \mathcal{A} \otimes_{E} E^{c}[G] \rightarrow$ $\mathcal{A}^{c}[G]$ is an isomorphism of $E$-algebras.

Proposition 4.5. Let $B$ be a $K$-building block over a Galois number $K$ field with group $G=$ $\operatorname{Gal}(K / \mathbb{Q})$. Let $\mathcal{D}=\operatorname{End}_{K}(B)$ and $E=Z(\mathcal{D})$. Then,

$$
\operatorname{End}_{\mathbb{Q}}\left(\operatorname{Res}_{K / \mathbb{Q}}(B)\right) \simeq \mathcal{D} \otimes_{E} E^{c_{B / K}}[G] .
$$

Proof. Call $A$ the variety $\operatorname{Res}_{K / \mathbb{Q}}(B)$. For each $s \in G$ fix a representative $\tilde{s}$ for $s$ in $G_{\mathbb{Q}}$ imposing that $\tilde{1}=1$. Let $\left\{\mu_{\sigma}\right\}_{\sigma \in G_{\mathbb{Q}}}$ be a locally constant set of isogenies compatible with $\operatorname{End}(B)$ defined over $K$ in which we have chosen $\mu_{1}$ to be the identity. We know that $A \sim_{K} \prod_{s \in G}{ }^{\tilde{s}} B$, and that by the universal property of the restriction of scalars functor $\operatorname{End}_{\mathbb{Q}}(A) \simeq \operatorname{Hom}_{K}(A, B)$. Hence,

$$
\operatorname{End}_{\mathbb{Q}}(A) \simeq \operatorname{Hom}_{K}(A, B) \simeq \prod_{s \in G} \operatorname{Hom}_{K}\left({ }^{\tilde{s}} B, B\right) \simeq \prod_{s \in G} \mathcal{D} \cdot \mu_{\tilde{s}}
$$

and we see that $\operatorname{End}_{\mathbb{Q}}(A)$ is a left $\mathcal{D}$-module of dimension $[K: \mathbb{Q}]$. We shall determine now its structure as an algebra. Define, for $s \in G, \lambda_{s}$ to be the endomorphism of $A$ which sends ${ }^{\tilde{t s}} B$ to ${ }^{\tilde{t}} B$ via ${ }^{\tilde{t}} \mu_{\tilde{s}}$. It is fixed by all elements in $G_{\mathbb{Q}}$ and so it is an endomorphism of $A$ defined over $\mathbb{Q}$. Since we forced $\tilde{1}$ to be 1 , we can identify $\lambda_{1}$ with the identity endomorphism of $\operatorname{End}_{\mathbb{Q}}(A)$.

We can embed $\mathcal{D}$ in $\operatorname{End}_{\mathbb{Q}}(A)$ by sending each $d \in \mathcal{D}$ to the morphism whose components are the diagonal maps ${ }^{\tilde{s}} d:{ }^{\tilde{s}} B \rightarrow{ }^{\tilde{s}} B$. Hence, we can multiply the $\lambda_{s}$ by elements $d$ in $\mathcal{D}$ in the following way, depending on whether we left or right multiply:

$$
\begin{aligned}
& d \circ \lambda_{s}:{ }^{\widetilde{t s}} B \stackrel{\tilde{t}_{\tilde{s}}}{\longrightarrow} \tilde{t} B \stackrel{\tilde{t}_{d}}{\longrightarrow}{ }^{\tilde{t}} B \\
& \lambda_{s} \circ d: \widetilde{t_{s}} B \stackrel{\tilde{t s}_{s}}{\longrightarrow}{ }^{\widetilde{t s}} B \stackrel{\tilde{t}_{\mu \tilde{s}}}{\longrightarrow}{ }^{\tilde{t}} B .
\end{aligned}
$$

By the compatibility of the isogenies it is clear that these two maps coincide, and therefore $d \circ \lambda_{s}=\lambda_{s} \circ d$. Also the compatibility of the isogenies gives us the formula $\lambda_{s} \circ \lambda_{t}=c_{B / K}(s, t) \circ \lambda_{s t}$. That is, multiplication in $\operatorname{End}_{\mathbb{Q}}(A)$ is given in terms of this basis by formulas (4.1) with cocycle $c_{B / K}$, so that this algebra is isomorphic to $\mathcal{D}^{c_{B / K}}[G]$.

\section{Strongly MOdUlar ABELIAN VARIETIES}

Let $B$ be a $K$-building block over a Galois number field $K$ with Galois group $G=\operatorname{Gal}(K / \mathbb{Q})$. Let $\mathcal{D}=\operatorname{End}_{K}(B), E=Z(\mathcal{D})$, and $t$ the index of $\mathcal{D}$. Recall that in the previous section we have associated to $B / K$ a cohomology class $\left[c_{B / K}\right] \in H^{2}\left(G, E^{*}\right)$. In this section we characterize when $B$ is strongly modular over $K$ in terms of that class. 
Lemma 5.1. Let $B$ be a $K$-building block over a Galois number field $K$. If $A=\operatorname{Res}_{K / \mathbb{Q}}(B)$ is an abelian variety of $\mathrm{GL}_{2}$-type, then

$$
A \sim_{\mathbb{Q}} A_{1}^{t} \times \cdots \times A_{n}^{t}
$$

with the $A_{i}$ pairwise non-isogenous $\mathbb{Q}$-simple abelian varieties of $\mathrm{GL}_{2}$-type.

Proof. A priori we know that

$$
A \sim_{\mathbb{Q}} A_{1}^{r_{1}} \times \cdots \times A_{n}^{r_{n}}
$$

for some $r_{i}>0$ and with the $A_{i}$ being non-isogenous $\mathbb{Q}$-simple abelian varieties of $\mathrm{GL}_{2}$-type. If we set $E_{i}=\operatorname{End}_{\mathbb{Q}}\left(A_{i}\right)$ then $\operatorname{End}_{\mathbb{Q}}(A) \simeq \mathrm{M}_{r_{1}}\left(E_{1}\right) \times \cdots \times \mathrm{M}_{r_{n}}\left(E_{n}\right)$.

First we show that each $r_{i}$ is at least $t$. If $t=1$ this is clear, so we suppose now that $t=2$. There is an injection of algebras $\operatorname{End}_{K}(B) \hookrightarrow \operatorname{End}_{\mathbb{Q}}(A)$, and so $\operatorname{End}_{K}(B)$ injects into each simple component $M_{r_{i}}\left(E_{i}\right)$ of $\operatorname{End}_{\mathbb{Q}}(A)$. If $t=2$ then $\operatorname{End}_{K}(B)$ is non-commutative, and so each $r_{i}$ must be at least 2 .

Now we show that each $r_{i}$ is in fact equal to $t$. On the one hand we know, by the universal property of the restriction of scalars, that $\operatorname{End}_{\mathbb{Q}}(A) \simeq \operatorname{Hom}_{K}\left(A_{K}, B\right)$, and using that $A_{K} \sim_{K}$ $\prod_{s \in \operatorname{Gal}(K / \mathbb{Q})}{ }^{s} B$ we have that

$$
\operatorname{End}_{\mathbb{Q}} A \simeq \operatorname{Hom}_{K}\left(A_{K}, B\right) \simeq \operatorname{Hom}_{K}\left(\prod^{s} B, B\right) \simeq \bigoplus_{s \in \operatorname{Gal}(K / \mathbb{Q})} \operatorname{Hom}_{K}\left({ }^{s} B, B\right) .
$$

Since $B$ is a $K$-building block each ${ }^{s} B$ is $K$-isogenous to $B$, and so we have an isomorphism of $\mathcal{D}$-modules $\operatorname{Hom}_{K}\left({ }^{s} B, B\right) \simeq \mathcal{D}$. Since $\mathcal{D}$ is a $\mathbb{Q}$-vector space of dimension $t^{2}[E: \mathbb{Q}]=t \operatorname{dim} B$ one obtains $\operatorname{dim}_{\mathbb{Q}} \operatorname{End}_{\mathbb{Q}}(A)=|G| t \operatorname{dim} B=t \operatorname{dim} A$.

On the other hand, we can use expression (5.1) to calculate the same dimension. We have shown that $r_{i} \geqslant t$ for all $i$. Suppose that for some $i$ we had $r_{i}>t$. Then we would find that

$$
\begin{aligned}
\operatorname{dim}_{\mathbb{Q}} \operatorname{End}_{\mathbb{Q}} A & =r_{1}^{2} \operatorname{dim} A_{1}+\cdots+r_{n}^{2} \operatorname{dim} A_{n}>t r_{1} \operatorname{dim} A_{1}+\cdots+t r_{n} \operatorname{dim} A_{n}= \\
& =t\left(r_{1} \operatorname{dim} A_{1}+\cdots+r_{n} \operatorname{dim} A_{n}\right)=t \operatorname{dim} A,
\end{aligned}
$$

which is a contradiction with the first calculation we made.

Lemma 5.2. Let $B$ be a $K$-building block over a Galois number field with $G=\operatorname{Gal}(K / \mathbb{Q})$. Then $B$ is strongly modular if and only if the algebra $E^{c_{B / K}}[G]$ is commutative.

Proof. First suppose that $E^{c_{B / K}}[G]$ is commutative. Then it is a product of fields so that $E^{c_{B / K}}[G]=\prod E_{i}$. Call $A$ the variety $\operatorname{Res}_{K / \mathbb{Q}}(B)$. By Proposition 4.5 we know that

$$
\operatorname{End}_{\mathbb{Q}}(A) \simeq \mathcal{D} \otimes_{E} E^{c_{B} / K}[G] \simeq \prod \mathcal{D} \otimes_{E} E_{i},
$$

with $\mathcal{D} \otimes_{E} E_{i}$ a central simple $E_{i}$-algebra with index $t_{i}$ dividing $t$. Corresponding to this decomposition of $\operatorname{End}_{\mathbb{Q}}(A)$ there is a decomposition of $A$ up to $\mathbb{Q}$-isogeny: $A \sim_{\mathbb{Q}} \prod A_{i}$, and $\operatorname{End}_{\mathbb{Q}}\left(A_{i}\right) \simeq \mathcal{D} \otimes_{E} E_{i}$. As $A_{K} \simeq \prod^{s} B \sim_{K} B^{|G|}$, each $A_{i}$ is $K$-isogenous to $B^{n_{i}}$ for some $n_{i}$. We claim that $n_{i}$ equals $\left[E_{i}: E\right]$. To prove the claim, first we observe that the natural inclusion $\operatorname{End}_{\mathbb{Q}}\left(A_{i}\right) \hookrightarrow \operatorname{End}_{K}\left(A_{i}\right)$ gives an injective morphism $\mathcal{D} \otimes_{E} E_{i} \hookrightarrow \mathrm{M}_{n_{i}}(\mathcal{D})$. Looking at the reduced degrees of these algebras over $E$ we see that $t\left[E_{i}: E\right] \leqslant t n_{i}$, and then $\left[E_{i}: E\right] \leqslant n_{i}$. To see the equality, we can use that on the one hand, as $\operatorname{End}_{\mathbb{Q}}(A) \simeq \bigoplus_{s \in G} \operatorname{Hom}\left({ }^{s} B, B\right) \simeq \bigoplus_{s \in G} \mathcal{D}$ we have that

$$
\left[\operatorname{End}_{\mathbb{Q}}(A): E\right]=|G| t^{2}=t^{2} \sum n_{i}
$$

But, on the other hand we have that

$$
\left[\operatorname{End}_{\mathbb{Q}}(A): E\right]=\left[\mathcal{D} \otimes_{E} \prod E_{i}: E\right]=t^{2} \sum\left[E_{i}: E\right],
$$


and this gives that $\left[E_{i}: E\right]=n_{i}$.

Returning to the proof of the lemma, since $\operatorname{End}_{\mathbb{Q}}\left(A_{i}\right) \simeq \mathcal{D} \otimes_{E} E_{i}$ is a central simple algebra of index $t_{i} \mid t$, there exists a division $E_{i}$-algebra $\mathcal{D}_{i}$ of index $t_{i}$ acting on the differentials of $A_{i}$. The space of differentials of $A_{i}$ is a $\mathbb{Q}$-vector space of dimension equal to the dimension of $A_{i}$, and so we have that $\left[\mathcal{D}_{i}: \mathbb{Q}\right] \mid \operatorname{dim} A_{i}$. But $\left[\mathcal{D}_{i}: \mathbb{Q}\right]=t_{i}^{2}\left[E_{i}: E\right][E: \mathbb{Q}]$ and $\operatorname{dim} A_{i}=n_{i} \operatorname{dim} B=$ $n_{i} t[E: \mathbb{Q}]=t\left[E_{i}: E\right][E: \mathbb{Q}]$, because $n_{i}=\left[E_{i}: E\right]$. This means that

$$
t_{i}^{2}\left[E_{i}: E\right][E: \mathbb{Q}] \mid t\left[E_{i}: E\right][E: \mathbb{Q}]
$$

so $t_{i}^{2} \mid t$, which implies $t_{i}=1$. This means that $\mathcal{D} \otimes_{E} E_{i} \simeq \mathrm{M}_{t}\left(E_{i}\right)$, and therefore $A_{i} \sim_{\mathbb{Q}}\left(A_{i}^{\prime}\right)^{t}$, for some abelian variety $A_{i}^{\prime}$ with $\operatorname{End}_{\mathbb{Q}}\left(A_{i}^{\prime}\right) \simeq E_{i}$. Finally, $A_{i}^{\prime} \sim_{K} B^{n_{i} / t}$, which gives that

$$
\left[E_{i}: \mathbb{Q}\right]=n_{i}[E: \mathbb{Q}]=\frac{n_{i}}{t} t[E: \mathbb{Q}]=\frac{n_{i}}{t} \operatorname{dim} B=\operatorname{dim} A_{i}^{\prime}
$$

showing that each $A_{i}^{\prime}$ is a variety of $\mathrm{GL}_{2}$-type.

In order to prove the other implication, by the previous lemma we can suppose that $A \sim \mathbb{Q}$ $A_{1}^{t} \times \cdots \times A_{n}^{t}$, and as a consequence that

$$
\operatorname{End}_{\mathbb{Q}}(A) \simeq \mathrm{M}_{t}\left(E_{1}\right) \times \cdots \times \mathrm{M}_{t}\left(E_{n}\right)
$$

where the notation is the same as in the first part of the proof. On the other hand,

$$
\operatorname{End}_{\mathbb{Q}}(A) \simeq \mathcal{D} \otimes_{E} E^{c_{K}}[G]=\mathcal{D} \otimes_{E} \prod \mathrm{M}_{r_{i}}\left(C_{i}\right)
$$

where the $C_{i}$ are division algebras. But (5.3) forces $r_{i}=1$ and $C_{i} \simeq E_{i}$ for each $i$.

Now we state our main result, giving a characterization of strong modularity.

Theorem 5.3. Let $K$ be a Galois number field and let $B / K$ be a $K$-simple abelian variety. Then $B$ is strongly modular over $K$ if and only if it is a $K$-building block, the extension $K / \mathbb{Q}$ is abelian, and $\left[c_{B / K}\right]$ belongs to the subgroup $\operatorname{Ext}\left(G, E^{*}\right) \subseteq H^{2}\left(G, E^{*}\right)$ consisting of symmetric cocycle classes.

Proof. By Corollary 4.3 being a $K$-building block is a necessary condition, and in that case the previous lemma says that being strongly modular is equivalent to the fact that the algebra $E^{c_{B / K}}[G]$ is commutative. A twisted group algebra $E^{c}[G]$ is commutative if and only if the group $G$ is abelian and the cocycle $c$ is symmetric.

Strongly modular simple varieties. The previous theorem shows that strong modularity puts very restrictive conditions on varieties. In what follows, we consider the setting in which $B / \overline{\mathbb{Q}}$ is a $\overline{\mathbb{Q}}$-building block. Given a Galois number field $K$, using Theorem 5.3 we want to give necessary and sufficient conditions to guarantee the existence, in the $\overline{\mathbb{Q}}$-isogeny class of $B$, of some variety which is completely defined over $K$ and strongly modular over $K$.

For that, let $B$ be a $\overline{\mathbb{Q}}$-building block and let $\mathcal{D}=\operatorname{End}(B)$. The center $F=Z(\mathcal{D})$ is a totally real number field and $\mathcal{D}$ is either equal to $F$, in which case $t=1$ and $[F: \mathbb{Q}]=\operatorname{dim} B$, or it is a totally indefinite quaternion algebra over $F$, with $t=2$ and $[F: \mathbb{Q}]=\frac{1}{2} \operatorname{dim} B$. Let $\xi=\left[c_{B}\right] \in H^{2}\left(G_{\mathbb{Q}}, F^{*}\right)$ be the cohomology class attached to $B$.

We fix an embedding $F \hookrightarrow \overline{\mathbb{Q}}$. By a theorem of Tate it is known that the group $H^{2}\left(G_{\mathbb{Q}}, \overline{\mathbb{Q}}^{*}\right)$ is trivial (here $G_{\mathbb{Q}}$ acts trivially in $\overline{\mathbb{Q}}$ ), so there exist continuous maps $\alpha: G_{\mathbb{Q}} \rightarrow \overline{\mathbb{Q}}^{*}$ such that $c_{B}(\sigma, \tau)=\alpha(\sigma) \alpha(\tau) \alpha(\sigma \tau)^{-1}$ for all $\sigma, \tau \in G_{\mathbb{Q}}$; two such maps differ by a Galois character. The map $\bar{\alpha}: G_{\mathbb{Q}} \rightarrow \overline{\mathbb{Q}}^{*} / F^{*}$ obtained viewing the values of $\alpha$ modulo elements of $F^{*}$ is a morphism; let $K_{\alpha}$ denote the fixed field of its kernel, which is an abelian extension of $\mathbb{Q}$. Using the identity (3.3) we see that the map $\varepsilon_{\alpha}(\sigma)=\alpha(\sigma)^{2} / \delta\left(\mu_{\sigma}\right)$ is a Galois character $G_{\mathbb{Q}} \rightarrow \overline{\mathbb{Q}}^{*}$; two such characters differ by the square of a Galois character. Let $K_{\varepsilon_{\alpha}}$ be the fixed field of ker $\varepsilon_{\alpha}$; the fact that $\delta\left(\mu_{\sigma}\right)$ 
is real implies that $K_{\varepsilon_{\alpha}} \subseteq K_{\alpha}$. Let $E_{\alpha}=F\left(\{\alpha(\sigma)\}_{\sigma \in G_{\mathbb{Q}}}\right)$ be the number field generated over $F$ by the values of $\alpha$; from the identity defining $\varepsilon_{\alpha}(\sigma)$ it easily follows that $E_{\alpha} / F$ is an abelian extension. Even though the splitting maps $\alpha$ depend on the cocycle $c_{B}$ (or, what is the same, on a system of isogenies between conjugates of $B$ ) the morphisms $\bar{\alpha}$, the fields $K_{\alpha}$ and $E_{\alpha}$, and the characters $\varepsilon_{\alpha}$ do not depend on that choice. We will call the maps $\alpha$ splitting maps, the fields $K_{\alpha}$ splitting fields, and the characters $\varepsilon_{\alpha}$ splitting characters for the building block $B$. The isogeny class of a building block determines a set of morphisms $\bar{\alpha} \in \operatorname{Hom}\left(G_{\mathbb{Q}}, \overline{\mathbb{Q}}^{*} / F^{*}\right)$ that is an orbit by the action of the group of Galois characters $\operatorname{Hom}\left(G_{\mathbb{Q}}, \overline{\mathbb{Q}}^{*}\right)$, and a set of splitting characters $\varepsilon_{\alpha} \in \operatorname{Hom}\left(G_{\mathbb{Q}}, \overline{\mathbb{Q}}^{*}\right)$ that is an orbit by the action of the subgroup of squares $\operatorname{Hom}\left(G_{\mathbb{Q}}, \overline{\mathbb{Q}}^{*}\right)^{2}$.

For every Galois character $\varepsilon: G_{\mathbb{Q}} \rightarrow \overline{\mathbb{Q}}^{*}$ choose square roots of its values and define

$$
c_{\varepsilon}(\sigma, \tau)=\sqrt{\varepsilon(\sigma)} \sqrt{\varepsilon(\tau)} \sqrt{\varepsilon(\sigma \tau)}^{-1} .
$$

This is a 2 -cocycle on $G_{\mathbb{Q}}$ with values in $\{ \pm 1\}$. Its cohomology class $\left[c_{\varepsilon}\right] \in H^{2}\left(G_{\mathbb{Q}},\{ \pm 1\}\right) \simeq$ $\mathrm{Br}_{2}(\mathbb{Q})$ gives the obstruction to the existence of a square root of $\varepsilon$. If two characters $\varepsilon, \varepsilon^{\prime}$ differ by the square of a character, then $\left[c_{\varepsilon}\right]=\left[c_{\varepsilon^{\prime}}\right]$. If $\xi=\left[c_{B}\right] \in H^{2}\left(G_{\mathbb{Q}}, F^{*}\right)$ is the class attached to a building block $B$, then $\xi_{ \pm}=\left[c_{\varepsilon}\right]$ with $\varepsilon$ any splitting character for $B$ (see [18, Theorem 2.6]).

Theorem 5.4. Let $B / \overline{\mathbb{Q}}$ be a building block and let $K / \mathbb{Q}$ an abelian extension. There exists an abelian variety isogenous to $B$ that is completely defined and strongly modular over the field $K$ if and only if $K$ contains a splitting field for $\left[c_{B}\right]$.

Proof. The proof is essentially the same given in [16, Proposition 5.2] for the case of $\mathbb{Q}$-curves.

Suppose that $K$ contains the splitting field $K_{\alpha}$ corresponding to some splitting map $\alpha$. For every element $s \in \operatorname{Gal}(K / \mathbb{Q})$ choose an element $\alpha(s)$ as any of the values $\alpha(\sigma)$ for $\sigma \in G_{\mathbb{Q}}$ an automorphism restricting to $s$, and define $c(s, t)=\alpha(s) \alpha(t) \alpha(s t)^{-1}$. Then $[c]$ is an element of $H^{2}\left(K / \mathbb{Q}, F^{*}\right)$ whose inflation equals $\left[c_{B}\right]$. By Proposition 3.4 there exists an abelian variety $B_{0}$ isogenous to $B$ that is completely defined over the field $K$ and with $\left[c_{B_{0} / K}\right]=[c]$. By construction the cocycle $c$ is symmetric, hence $\left[c_{B_{0} / K}\right] \in \operatorname{Ext}\left(K / \mathbb{Q}, F^{*}\right)$ and by Theorem 5.3 the variety $B_{0}$ is strongly modular over $K$.

Conversely, assume that there is a variety isogenous to $B$ that is strongly modular over the field $K$. Let $c_{B_{0} / K}$ be a cocycle on $G=\operatorname{Gal}(K / \mathbb{Q})$ attached to this variety. Then by Theorem 5.3 the algebra $F^{c_{B_{0}} / K}[G]$ is commutative. Hence the $\overline{\mathbb{Q}}$-algebra $\overline{\mathbb{Q}}^{c_{B_{0}} / K}[G]=\overline{\mathbb{Q}} \otimes_{F} F^{c_{B_{0}} / K}[G]$ is also commutative, and by a property of twisted group algebras over algebraically closed fields (cf. [10, Chapter 2, Corollary 2.5]) it follows that the image of the class $\left[c_{B_{0} / K}\right]$ in the Schur multiplier group $H^{2}\left(G, \overline{\mathbb{Q}}^{*}\right)$ is trivial. Hence there exists a map $s \mapsto \alpha(s): G \rightarrow \overline{\mathbb{Q}}^{*}$ such that $c_{B_{0} / K}(s, t)=\alpha(s) \alpha(t) \alpha(s t)^{-1}$ and its inflation to the group $G_{\mathbb{Q}}$ is a splitting map for the variety that factors through the group $G$, hence $K_{\alpha} \subseteq K$.

\section{QM JACOBIAN SURFACES}

In this section we illustrate the previous general results with applications to the study of concrete abelian surfaces with quaternionic multiplication. The surfaces we will be dealing with are obtained as Jacobians of a family of genus two curves defined in [9]. We will use some results on their arithmetic that appear in [1] to compute the cocycles needed for the characterization of their strong modularity, and for the computation of $\mathbb{Q}$-endomorphism algebras of their restriction of scalars.

Since we will need quadratic twists later, we begin with a technical lemma describing the effect of such a twist in the cohomology classes of interest. For every abelian variety $B / K$ over a number field $K$ and element $\gamma \in K^{*}$, let $B_{\gamma}$ denote the $K(\sqrt{\gamma})$-quadratic twist of the variety 
$B$ over $K$. In the standard classification of twists by elements of the first Galois cohomology group with values in the automorphism group of the object, this variety corresponds to the homomorphism in $H^{1}\left(G_{K},\{ \pm 1\}\right)$ whose kernel has $K(\sqrt{\gamma})$ as fixed field, which is given by the formula $\sigma \mapsto \sqrt{\gamma} / \sqrt{\gamma}$. Note that here we interpret \pm 1 as automorphisms of $B$. In other words, $B_{\gamma}$ is the abelian variety determined up to $K$-isomorphism by the fact that there exists an isomorphism $\phi: B_{\gamma} \rightarrow B$ defined over $K(\sqrt{\gamma})$ such that $\phi{ }^{\sigma}{ }^{\sigma} \phi^{-1}={ }^{\sigma} \sqrt{\gamma} / \sqrt{\gamma}$ for every $\sigma \in G_{K}$.

For hyperelliptic Jacobians the quadratic twists are easily computed: if $C$ is a hyperelliptic curve defined by the equation $Y^{2}=F(X)$ then for every $\gamma \in K^{*}$ the equation $\gamma Y^{2}=F(X)$ defines an hyperelliptic curve that is the $K(\sqrt{\gamma})$-quadratic twist of $C$ over $K$. The Jacobian $\operatorname{Jac}\left(\mathrm{B}_{\gamma}\right)$ is the $K(\sqrt{\gamma})$-quadratic twist of the abelian variety $\operatorname{Jac}(B)$ over $K$.

Lemma 6.1. Let $B / K$ be a $\mathbb{Q}$-variety completely defined over a Galois number field $K$, and let $\gamma \in K^{*}$. The twist $B_{\gamma}$ is completely defined over $K$ if and only if the field $K(\sqrt{\gamma})$ is Galois over $\mathbb{Q}$. In this case, $\left[c_{B / K}\right]$ and $\left[c_{B_{\gamma} / K}\right]$ differ by the cohomology class in $H^{2}(\operatorname{Gal}(K / \mathbb{Q}),\{ \pm 1\})$ corresponding to the group extension given by the exact sequence

$$
1 \longrightarrow \operatorname{Gal}(K(\sqrt{\gamma}) / K) \simeq\{ \pm 1\} \longrightarrow \operatorname{Gal}(K(\sqrt{\gamma}) / \mathbb{Q}) \longrightarrow \operatorname{Gal}(K / \mathbb{Q}) \longrightarrow 1 .
$$

In particular, quadratic twisting affects only the sign components of the cohomology classes and leaves the degree components unchanged.

Proof. Note that the cohomology classes $\left[c_{B / K}\right]$ and $\left[c_{B_{\gamma} / K}\right]$ we want to compare take values in groups $F^{*}$ consisting of automorphisms of the varieties. The cohomology class attached to the group extension of the statement takes values in the group $\{ \pm 1\}$, which must be identified with a subgroup of $F^{*}$ by the (canonical) identification of its elements as automorphisms of the variety.

Let $\phi: B_{\gamma} \rightarrow B$ be the isomorphism corresponding to the twist. Then $\phi^{-1}$ is an isomorphism giving $B$ as the $K(\sqrt{\gamma})$-twist of $B_{\gamma}$ and for every $\sigma \in G_{\mathbb{Q}}$ the map ${ }^{\sigma} \phi:{ }^{\sigma}\left(B_{\gamma}\right) \rightarrow{ }^{\sigma} B$ is an isomorphism giving ${ }^{\sigma}\left(B_{\gamma}\right)$ as the $K(\sqrt{\sigma} \gamma)$-twist of ${ }^{\sigma} B$. Every isogeny $\nu_{\sigma}:{ }^{\sigma} B_{\gamma} \rightarrow B_{\gamma}$ compatible with $\operatorname{End}(B)$ is of the form $\nu_{\sigma}=\phi^{-1} \circ \mu_{\sigma}{ }^{\circ}{ }^{\sigma} \phi$ for an isogeny $\mu_{\sigma}:{ }^{\sigma} B \rightarrow B$ compatible with $\operatorname{End}(B)$, which by hypothesis is defined over $K$. For $\tau \in G_{K}$ one has

$$
{ }^{\tau} \nu_{\sigma}={ }^{\tau} \phi^{-1}{ }^{\tau} \mu_{\sigma} \circ \tau \sigma \phi={ }^{\tau} \phi^{-1} \circ \phi \circ \nu_{\sigma} \circ{ }^{\sigma} \phi^{-1}{ }^{\tau \sigma} \phi=\frac{\sqrt{\gamma}}{\tau \sqrt{\gamma}}{ } \nu_{\sigma} \circ \frac{\sqrt{\sigma_{\gamma}}}{\tau \sqrt{\sigma \gamma}},
$$

which equals $\nu_{\sigma}$ if and only if the two other maps, each equal to \pm 1 , coincide. But

$$
\frac{\sqrt{\gamma}}{\tau \sqrt{\gamma}}=\frac{\sqrt{\sigma_{\gamma}}}{\tau \sqrt{\sigma_{\gamma}}} \Leftrightarrow \frac{\sqrt{\gamma}}{\sqrt{\sigma_{\gamma}}}=\frac{\tau \sqrt{\gamma}}{\tau \sqrt{\sigma_{\gamma}}} \Leftrightarrow \quad \Leftrightarrow \quad \text { fixes } \quad \sqrt{\sigma \gamma} / \sqrt{\gamma} \text {. }
$$

Hence the isogeny $\nu_{\sigma}$ is defined over $K$ if and only if $\sqrt{\sigma} \gamma / \sqrt{\gamma} \in K$, and this condition is satisfied for every $\sigma \in G_{\mathbb{Q}}$ exactly when the extension $K(\sqrt{\gamma}) / \mathbb{Q}$ is Galois.

Now assume the condition is satisfied. For each $s \in \operatorname{Gal}(K / \mathbb{Q})$ fix a lift $\tilde{s}$ of $s$ in $\operatorname{Gal}(K(\sqrt{\gamma}) / \mathbb{Q})$. Then

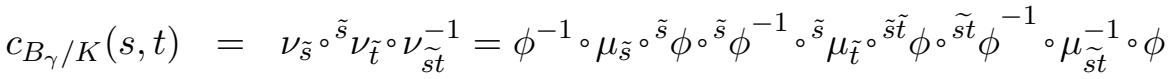

$$
\begin{aligned}
& =\phi^{-1} \circ \mu_{\tilde{s}} \circ{ }^{\tilde{s}} \mu_{\tilde{t}^{\circ}} \widetilde{s t}\left(\tilde{s t}^{-1} \tilde{s} \tilde{t} \phi \circ \phi^{-1}\right) \circ \mu_{\tilde{s t}}^{-1} \circ \phi \\
& =\phi^{-1} \circ \mu_{\tilde{s}} \circ{ }^{\tilde{s}} \mu_{\tilde{t}} \circ \mu_{\tilde{s t}}^{-1} \circ \phi \circ\left(\widetilde{s t}^{-1} \tilde{s} \tilde{t} \phi \circ \phi^{-1}\right)
\end{aligned}
$$

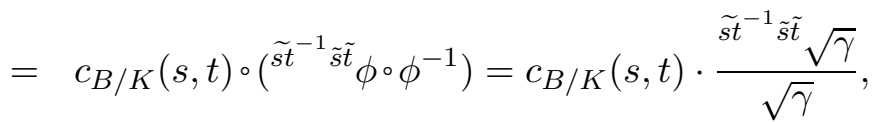


and the factor in the right is a cocycle associated to the group extension (6.1).

We now recall some notation and results from [1]. If $a, b \in \mathbb{Q}$ we denote $(a, b)_{\mathbb{Q}}$ the quaternion algebra over $\mathbb{Q}$ generated by $\imath, \jmath$ with $\imath^{2}=a, \jmath^{2}=b$ and $\imath+\jmath=0$. Let $\mathcal{Q}_{6}=(2,3)_{\mathbb{Q}}$ be the quaternion algebra of discriminant 6 over $\mathbb{Q}$, and let $\mathcal{O}=\mathbb{Z}[\imath,(1+\jmath) / 2]$, which is a maximal order of $\mathcal{Q}_{6}$. We also define the subrings $R_{2}=\mathbb{Z}[\imath] \simeq \mathbb{Z}[\sqrt{2}], R_{3}=\mathbb{Z}[\jmath+\imath \jmath] \simeq \mathbb{Z}[\sqrt{-} 3]$ and $R_{6}=\mathbb{Z}[\mu] \simeq \mathbb{Z}[\sqrt{6}]$, where $\mu=2 \jmath+\imath$. A curve $C$ is said to be a $Q M$-curve with respect to $\mathcal{O}$ if $\mathcal{O}$ can be embedded into the endomorphism ring of its Jacobian. If $(B, \rho) / \overline{\mathbb{Q}}$ is a polarized abelian variety and $R$ is a subring of $\operatorname{End}(B)$, the field of moduli $k_{R}$ is defined to be the smallest number field such that for any $\sigma \in \operatorname{Gal}\left(\overline{\mathbb{Q}} / k_{R}\right)$ there exists an isomorphism $\phi_{\sigma}:{ }^{\sigma} B \rightarrow B$ with $\phi_{\sigma}^{*}(\rho)={ }^{\sigma} \rho$ and such that $r \circ \phi_{\sigma}=\phi_{\sigma}{ }^{\circ} \sigma r$ for all $r \in R$. In other words, $k_{R}$ is the field of moduli of the object consisting of the polarized abelian variety $(B, \rho)$ together with the ring of endomorphisms $R \subseteq \operatorname{End}(B)$.

The family of surfaces we are going to consider is the following. For every algebraic number $j \in \overline{\mathbb{Q}}$ let $C_{j}$ be the genus 2 curve with equation

$$
\begin{aligned}
C_{j}: \quad Y^{2}= & (-4+3 \sqrt{-6 j}) X^{6}-12(27 j+16) X^{5}-6(27 j+16)(28+9 \sqrt{-6 j}) X^{4} \\
& +16(27 j+16)^{2} X^{3}+12(27 j+16)^{2}(28-9 \sqrt{-6 j}) X^{2} \\
& -48(27 j+16)^{3} X+8(27 j+16)^{3}(4+3 \sqrt{-6 j})
\end{aligned}
$$

Let $B_{j}=\operatorname{Jac}\left(C_{j}\right)$ be its Jacobian with the canonical principal polarization induced by $C_{j}$. Some properties of these objects proved in [1] are summarized in the following statement.

Theorem 6.2 (Baba-Granath).

- The curve $C_{j}$ has field of moduli $\mathbb{Q}(j)$. For every $\sigma \in G_{\mathbb{Q}(j)}$ such that $\sqrt{-6 j}=-\sqrt{-6 j}$ the map $(x, y) \mapsto\left(\frac{-2(27 j+16)}{x}, \frac{y(-2(27 j+16))^{3 / 2}}{x^{3}}\right)$ is an isomorphism ${ }^{\sigma} C_{j} \rightarrow C_{j}$.

- The field $\mathbb{Q}(\sqrt{-6 j}) k_{\mathcal{O}}$ is a field of definition of the endomorphisms of $B_{j}$.

- The curves $C_{j}$ are $Q M$-curves with respect to $\mathcal{O}$. Moreover, for all $j \in \mathbb{Q}$ but for 26 values, $\operatorname{End}^{0}\left(B_{j}\right) \simeq \mathcal{Q}_{6}$. Under this isomorphism the Rosati involution' attached to the canonical polarization of $B_{j}$ is given by $\varphi^{\prime}=\mu^{-1} \varphi^{*} \mu$, where ${ }^{*}$ indicates the canonical conjugation of $\mathcal{Q}_{6}$.

- The fields of moduli $k_{R}$ for the canonically polarized Jacobian $B_{j}$ and several rings of endomorphisms of interest are given in the following diagram:

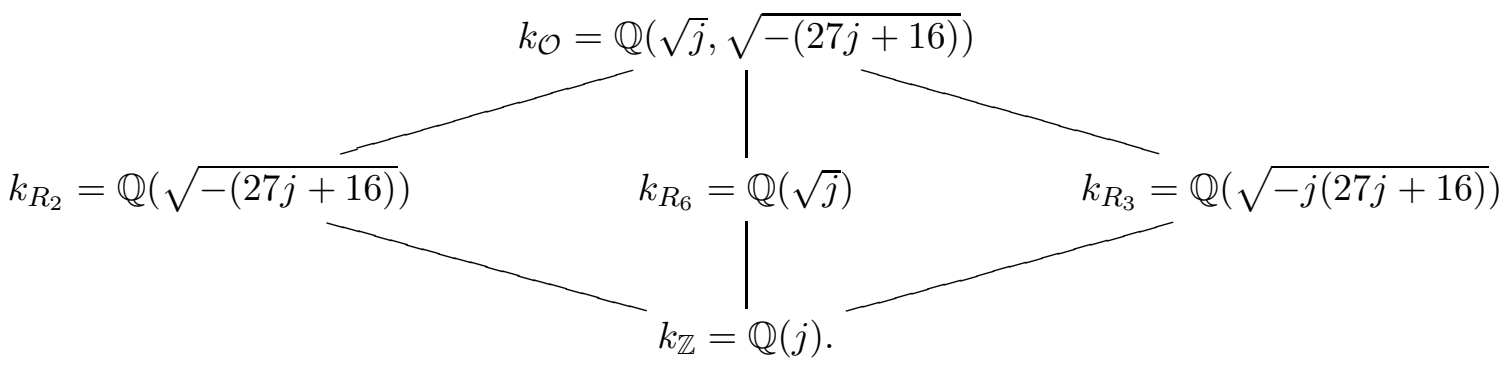

When $j \in \mathbb{Q}$ the abelian surfaces $B_{j}$ have the property that for every $\sigma \in G_{\mathbb{Q}}$ there exists an isomorphism $\phi_{\sigma}:{ }^{\sigma} B_{j} \rightarrow B_{j}$, but this isomorphism does not need to be compatible with $\operatorname{End}\left(B_{j}\right)$ (indeed, in general it is not compatible with $\operatorname{End}\left(B_{j}\right)$ ). However, if the algebra of endomorphisms of $B_{j}$ is isomorphic to $\mathcal{Q}_{6}$ then we can always find isogenies compatible with $\operatorname{End}\left(B_{j}\right)$. More generally, we have the following result. 
Proposition 6.3. Let $B / \overline{\mathbb{Q}}$ be an abelian variety whose algebra of endomorphisms is a central simple $\mathbb{Q}$-algebra. Let $\sigma \in G_{\mathbb{Q}}$. If ${ }^{\sigma} B$ and $B$ are isogenous then there exists an isogeny ${ }^{\sigma} B \rightarrow B$ compatible with $\operatorname{End}(B)$.

Proof. Call $\mathcal{D}$ the endomorphism algebra of $B$, and let $\phi_{\sigma}:{ }^{\sigma} B \rightarrow B$ be an isogeny. The map $\varphi \mapsto \phi_{\sigma}{ }^{\sigma} \varphi^{\circ} \phi_{\sigma}^{-1}$ is a $\mathbb{Q}$-algebra automorphism of $\mathcal{D}$ since it fixes the center, which is $\mathbb{Q}$ by hypothesis. Then the Noether-Skolem Theorem implies that it is inner; that is, there exists an element $\psi_{\sigma} \in \mathcal{D}$ such that $\phi_{\sigma}{ }^{\circ} \varphi^{\circ} \phi_{\sigma}^{-1}=\psi_{\sigma}^{-1} \circ \varphi \circ \psi_{\sigma}$. Then the isogeny $\mu_{\sigma}=\psi_{\sigma} \circ \phi_{\sigma}$ is compatible with $\mathcal{D}$.

Hence, we see that if $j$ belongs to $\mathbb{Q}$ and the endomorphism algebra of $B_{j}$ is isomorphic to $\mathcal{Q}_{6}$, then $B_{j}$ is a building block completely defined over the field

$$
K=\mathbb{Q}(\sqrt{-6 j}, \sqrt{j}, \sqrt{-(27 j+16)}, \sqrt{-2(27 j+16)}) .
$$

From now on we assume that $\operatorname{End}\left(B_{j}\right) \otimes \mathbb{Q} \simeq \mathcal{Q}_{6}$. Now we aim to compute the cohomology class $\left[c_{B_{j}}\right]$. The degree component $\overline{\left[c_{B_{j}}\right]}$ belongs to $\operatorname{Hom}\left(G_{\mathbb{Q}}, \mathbb{Q}^{*} /\{ \pm 1\} \mathbb{Q}^{* 2}\right)$, and we use the following notation to indicate the elements of this group: for $t, d \in \mathbb{Q}^{*}$ we denote by $(t, d)_{P}$ the homomorphism that sends an element $\sigma \in G_{\mathbb{Q}}$ to $d \cdot\{ \pm 1\} \mathbb{Q}^{* 2}$ if $\sigma \sqrt{t}=-\sqrt{t}$ and has trivial image otherwise. An expression of the form $\left(t_{1}, d_{1}\right)_{P} \cdot\left(t_{2}, d_{2}\right)_{P} \cdot \ldots \cdot\left(t_{r}, d_{r}\right)_{P}$ denotes the product of such homomorphisms, and all elements in $\operatorname{Hom}\left(G_{\mathbb{Q}}, \mathbb{Q}^{*} /\{ \pm 1\} \mathbb{Q}^{* 2}\right)$ admit a (non-unique) expression of this kind.

Proposition 6.4. The degree and sign components of $\left[c_{B_{j}}\right]$ are given by

$$
\begin{gathered}
\overline{\left[c_{B_{j}}\right]}=(-(27 j+16), 3)_{P} \cdot(-j(27 j+16), 2)_{P}, \\
{\left[c_{B_{j}}\right]_{ \pm}=(-(27 j+16), 3)_{\mathbb{Q}} \cdot(-j(27 j+16), 2)_{\mathbb{Q}} \cdot(2,3)_{\mathbb{Q}} .}
\end{gathered}
$$

Proof. Recall that the degree component is the map $\sigma \mapsto \delta\left(\mu_{\sigma}\right) \bmod \{ \pm 1\} \mathbb{Q}^{* 2}$, where $\mu_{\sigma}$ is any isogeny $\mu_{\sigma}:{ }^{\sigma} B_{j} \rightarrow B_{j}$ compatible with $\operatorname{End}\left(B_{j}\right)$. If $\sigma \in \mathrm{Gal}\left(\overline{\mathbb{Q}} / k_{\mathcal{O}}\right)$, by the definition of $k_{\mathcal{O}}$ there exists an isomorphism $\phi_{\sigma}:{ }^{\sigma} B_{j} \rightarrow B_{j}$ compatible with $\operatorname{End}\left(B_{j}\right)$ such that $\phi_{\sigma}^{*}(\rho)={ }^{\sigma} \rho$, where $\rho$ is the polarization of $B_{j}$ given by $C_{j}$. Applying the definition of $\delta$ (see [15, p. 220]) we find that

$$
\delta\left(\phi_{\sigma}\right)=\phi_{\sigma}{ }^{\sigma} \rho^{-1} \circ \hat{\phi}_{\sigma} \circ \rho=\phi_{\sigma} \circ \phi_{\sigma}^{-1} \circ \rho^{-1} \circ \hat{\phi}_{\sigma}^{-1} \circ \hat{\phi}_{\sigma} \circ \rho=1
$$

Hence, the degree component is the inflation of a map defined in $\operatorname{Gal}\left(k_{\mathcal{O}} / \mathbb{Q}\right)$. Now, since $k_{\mathcal{O}}=k_{R_{2}} \cdot k_{R_{3}}$, we just need to compute $\delta\left(\mu_{\sigma}\right)$ for $\sigma \in \operatorname{Gal}\left(\overline{\mathbb{Q}} / k_{R_{d}}\right)$ for $d=2,3$.

Let $\sigma$ be an element in $\operatorname{Gal}\left(\overline{\mathbb{Q}} / k_{R_{d}}\right)$ that does not fix $k_{\mathcal{O}}$. Since $k_{R_{2}}=\mathbb{Q}(\sqrt{-(27 j+16)})$ and $k_{R_{3}}=\mathbb{Q}(\sqrt{-j(27 j+16)})$, in order to prove (6.3) we just need to see that $\delta\left(\mu_{\sigma}\right) \equiv d$ $\left(\bmod \mathbb{Q}^{* 2}\right)$. By the definition of $k_{R_{d}}$ there exists an isomorphism $\phi_{\sigma}:{ }^{\sigma} B \rightarrow B$ compatible with the endomorphisms in $R_{d}$, but not necessarily compatible with all the endomorphisms. However, we know from Proposition 6.3 that we can find $\psi_{\sigma} \in \mathcal{Q}_{6}$ such that $\mu_{\sigma}=\psi_{\sigma}{ }^{\circ} \phi_{\sigma}$ is an isogeny compatible with all the endomorphisms. Moreover, from the proof of this proposition we see that $\psi_{\sigma}$ is characterized by the property that

$$
\phi_{\sigma} \circ{ }^{\sigma} \varphi \circ \phi_{\sigma}^{-1}=\psi_{\sigma}^{-1} \circ \varphi \circ \psi_{\sigma}, \quad \text { for every } \varphi \in \mathcal{Q}_{6} .
$$

But if we take $\varphi \in R_{d}$, this particularizes to $\varphi=\psi_{\sigma}^{-1} \circ \varphi \circ \psi_{\sigma}$, so $\psi_{d}$ commutes with every element in $R_{d}$, which implies that $\psi_{d}$ belongs to $R_{d} \otimes \mathbb{Q}$. Hence, if we write $R_{d}=\mathbb{Z}\left[c_{d}\right]$, with $c_{2}=\imath$ and $c_{3}=\jmath+\imath \jmath$ we have that $\psi_{d}=a+b c_{d}$ for some $a, b \in \mathbb{Q}$. In fact, $b \neq 0$ because otherwise 
the isomorphism $\psi_{\sigma}$ would be compatible with all the endomorphisms of $B_{j}$, and this is not the case since we are assuming that $\sigma$ does not fix $k_{\mathcal{O}}$. Using the definition of $\delta\left(\mu_{\sigma}\right)$ we see that

$$
\begin{aligned}
\delta\left(\mu_{\sigma}\right) & =\delta\left(\psi_{\sigma} \circ \phi_{\sigma}\right)=\psi_{\sigma} \circ \phi_{\sigma} \circ \rho^{-1} \circ \widehat{\psi_{\sigma} \circ \phi_{\sigma}} \circ \rho=\psi_{\sigma} \circ \phi_{\sigma} \circ \phi_{\sigma}^{-1} \circ \rho^{-1} \circ \hat{\phi}_{\sigma}^{-1} \circ \hat{\phi}_{\sigma} \circ \hat{\psi}_{\sigma} \circ \rho \\
& =\psi_{\sigma} \circ \rho^{-1} \circ \hat{\psi}_{\sigma} \circ \rho=\psi_{\sigma} \circ \psi_{\sigma}^{\prime} .
\end{aligned}
$$

Now we know that if $\varphi \in \mathcal{Q}_{6}$ its Rosati involution is given by $\varphi^{\prime}=\mu^{-1} \varphi^{*} \mu$. Hence,

$$
\begin{aligned}
\delta\left(\mu_{\sigma}\right) & =\psi_{\sigma} \circ \psi_{\sigma}^{\prime}=\left(a+b c_{d}\right)\left(a+b c_{d}\right)^{\prime}=\left(a+b c_{d}\right) \mu^{-1}\left(a-b c_{d}\right) \mu \\
& =\left(a+b c_{d}\right)^{2}=a+d b^{2}+2 a b c_{d},
\end{aligned}
$$

and since $\delta\left(\mu_{\sigma}\right)$ must lie in $\mathbb{Q}^{*}$ and $b \neq 0$, we see that $a=0$ and $\delta\left(\mu_{\sigma}\right) \equiv d\left(\bmod \mathbb{Q}^{* 2}\right)$.

Now, to prove the identity (6.4) we use [18, Theorem 2.8], which gives a formula for the Brauer class of the endomorphism algebra of a building block. Specialized to our case, and having computed the degree component, this formula gives

$$
(2,3)_{\mathbb{Q}}=\left[c_{B_{j}}\right]_{ \pm} \cdot(-(27 j+16), 3)_{\mathbb{Q}} \cdot(-j(27 j+16), 2)_{\mathbb{Q}}
$$

and from this (6.4) follows.

A concrete example: $j=1 / 81$. Let us now consider the example corresponding to this value of the parameter; let $C=C_{j}$ and $B=\operatorname{Jac}(C)$. We remark that $B$ is $\mathrm{QM}$ and not $\mathrm{CM}$, i.e., $\operatorname{End}(B) \otimes \mathbb{Q} \simeq \mathcal{Q}_{6}$. Then $B$ is a building block completely defined over $K=\mathbb{Q}(\sqrt{-6}, \sqrt{-3})$ and it is strongly modular over $K$ if and only if $\left[c_{B / K}\right] \in \operatorname{Ext}\left(K / \mathbb{Q}, \mathbb{Q}^{*}\right)$; that is, if and only if it can be represented by a symmetric cocycle. In fact, since the degree component is always symmetric (over an abelian extension) we need to check this property only for the sign component $\left[c_{B / K}\right]_{ \pm} \in H^{2}(K / \mathbb{Q},\{ \pm 1\})$.

For $d=-3,-6$ we denote by $\varepsilon_{d}$ the non-trivial character $\varepsilon_{d}: \operatorname{Gal}(\mathbb{Q}(\sqrt{d}) / \mathbb{Q}) \rightarrow\{ \pm 1\}$. The group $H^{2}(K / \mathbb{Q},\{ \pm 1\})$ admits a basis as a $\mathbb{Z} / 2 \mathbb{Z}$-vector space consisting of the classes of three 2-cocycles that we call $c_{\varepsilon_{-6}}, c_{\varepsilon_{-3}}$ and $c_{-6,-3}$ (see for instance [17, Section 2] for the definition of these cocycles and their properties). Hence, we have that

$$
\left[c_{B / K}\right]_{ \pm}=\left[c_{\varepsilon_{-6}}\right]^{a} \cdot\left[c_{\varepsilon_{-3}}\right]^{b} \cdot\left[c_{-6,-3}\right]^{c}
$$

for some $a, b, c \in\{0,1\}$, and $\left[c_{B / K}\right]$ lies in $\operatorname{Ext}(K / \mathbb{Q},\{ \pm 1\})$ if and only if $c=0$. We know that $\operatorname{Inf}\left[c_{B / K}\right]_{ \pm}=\left[c_{B}\right]_{ \pm}$, which in this case turns out to be trivial by (6.4). Since $\operatorname{Inf}\left[c_{\varepsilon_{-6}}\right]=$ $(-6,-1)_{\mathbb{Q}}, \operatorname{Inf}\left[c_{\varepsilon_{-3}}\right]=(-3,-1)_{\mathbb{Q}}$ and $\operatorname{Inf}\left[c_{-6,-3}\right]=(-6,-3)_{\mathbb{Q}}$, the only possibilities are $a=$ $b=c=0$ or $a=b=1, c=0$. In both cases $c=0$, which implies that $\left[c_{B / K}\right]_{ \pm}$lies in $\operatorname{Ext}(K / \mathbb{Q},\{ \pm 1\})$ and therefore $B$ is strongly modular over $K$.

Let $\xi_{1}, \xi_{2} \in H^{2}\left(K / \mathbb{Q}, \mathbb{Q}^{*}\right)$ be the cohomology classes with degree component $\bar{\xi}_{1}=\bar{\xi}_{2}=$ $(-3,6)_{P}$ and sign component $\xi_{1 \pm}=1, \xi_{2 \pm}=\left[c_{\varepsilon_{-6}}\right] \cdot\left[c_{\varepsilon_{-3}}\right]$, and let $A=\operatorname{Res}_{K / \mathbb{Q}} B$. We have seen that either $\left[c_{B / K}\right]=\xi_{1}$ or $\left[c_{B / K}\right]=\xi_{2}$. By direct computation we see that

$$
\mathbb{Q}^{\xi_{1}}[G] \simeq \mathbb{Q}(\sqrt{6}) \times \mathbb{Q}(\sqrt{6}) \quad \text { and } \quad \mathbb{Q}^{\xi_{2}}[G] \simeq \mathbb{Q}(\sqrt{6}, \sqrt{-6})
$$

where $G=\operatorname{Gal}(K / \mathbb{Q})$. By Proposition 4.5 we see that if $\left[c_{B / K}\right]=\xi_{1}$ then $A \sim_{\mathbb{Q}} A_{g}^{2} \times A_{h}^{2}$ for some newforms $g$ and $h$ with $\operatorname{End}_{\mathbb{Q}}\left(A_{g}\right) \simeq \operatorname{End}_{\mathbb{Q}}\left(A_{h}\right) \simeq \mathbb{Q}(\sqrt{6})$. On the other hand, if $\left[c_{B / K}\right]=\xi_{2}$ then $A \sim_{\mathbb{Q}} A_{f}^{2}$ for some newform $f$ with $\operatorname{End}_{\mathbb{Q}}\left(A_{f}\right) \simeq \mathbb{Q}(\sqrt{6}, \sqrt{-6})$. If $\mathfrak{p}$ is a prime of $K$, let $L_{\mathfrak{p}}(B / K, T)$ be the numerator of the zeta function of the reduction of $B$ modulo $\mathfrak{p}$, and let

$$
L_{p}(B / K, T)=\prod_{\mathfrak{p} \mid p} L_{\mathfrak{p}}\left(B / K, T^{\mathrm{Np}}\right),
$$


which is in fact equal to $L_{p}(A / \mathbb{Q}, T)$. By counting points in the reduction of $B$ modulo primes of $K$ we have computed some of these local factors:

\begin{tabular}{|l|l|}
\hline$p$ & $L_{p}(B / K, T)^{-1}=L_{p}(A / \mathbb{Q}, T)^{-1}$ \\
\hline 5 & $\left(1-4 T^{2}+5^{2} T^{4}\right)^{4}$ \\
\hline 7 & $\left(1-2 T+7 T^{2}\right)^{8}$ \\
\hline 11 & $\left(1-16 T^{2}+11^{2} T^{4}\right)^{4}$ \\
\hline 13 & $\left(1-25 T^{2}+13^{2} T^{4}\right)^{4}$ \\
\hline 17 & $\left(1-20 T^{2}+17^{2} T^{4}\right)^{4}$ \\
\hline 19 & $\left(1-37 T^{2}+19^{2} T^{4}\right)^{4}$ \\
\hline 23 & $\left(1+40 T^{2}+23^{2} T^{4}\right)^{4}$ \\
\hline 29 & $\left(1-34 T^{2}+29^{2} T^{4}\right)^{4}$ \\
\hline 31 & $\left(1-T+31 T^{2}\right)^{8}$ \\
\hline 37 & $\left(1-10 T^{2}+37^{2} T^{4}\right)^{4}$ \\
\hline 41 & $\left(1+58 T^{2}+41^{2} T^{4}\right)^{4}$ \\
\hline
\end{tabular}

Some of these factors are of the form $\left(1+e_{p} T^{2}+p^{2} T^{4}\right)^{4}$, and if we had $A \sim_{\mathbb{Q}} A_{g}^{2} \times A_{h}^{2}$ for some newforms $g=\sum b_{n} q^{n}$ and $h=\sum c_{n} q^{n}$ this would imply that

$$
1+e_{p} T^{2}+p^{2} T^{4}=\left(1-b_{p} T+p T^{2}\right)\left(1-{ }^{\sigma} b_{p} T+p T^{2}\right)
$$

being $\sigma$ the non-trivial automorphism of $\mathbb{Q}(\sqrt{6}) / \mathbb{Q}$. A similar relation would hold for the coefficients $c_{p}$. But this relation implies that $b_{p}^{2}=c_{p}^{2}=2 p-e_{p}$, which is impossible for the computed values of $e_{p}$ because then the coefficients $b_{p}$ and $c_{p}$ would not lie in $\mathbb{Q}(\sqrt{6})$. Therefore, the actual cohomology class is $\left[c_{B / K}\right]=\xi_{2}$ and $A \sim_{\mathbb{Q}} A_{f}^{2}$ for some newform $f=\sum a_{n} q^{n}$ with the $a_{n}$ generating $\mathbb{Q}(\sqrt{6}, \sqrt{-6})$. However, Proposition 3.3 tells us that there also exists a variety in the $\overline{\mathbb{Q}}$-isogeny class of $B$ completely defined over $K$ and with cohomology class $\xi_{1}$. We will find such a variety as the Jacobian of a quadratic twist of $C$.

Let $\gamma=2-\sqrt{2}$. The extension $K(\sqrt{\gamma}) / \mathbb{Q}$ is Galois, and an easy computation shows that the cohomology class associated to (6.1) in this particular case is $\left[c_{\varepsilon_{-6}}\right] \cdot\left[c_{\varepsilon_{-3}}\right]$. Hence, the variety $B_{\gamma}$ is completely defined over $K$ and $\left[c_{B_{\gamma} / K}\right]=c_{B / K} \cdot\left[c_{\varepsilon_{-6}}\right] \cdot\left[c_{\varepsilon_{-3}}\right]=\xi_{1}$. Arguing as before we see that $A_{\gamma}=\operatorname{Res}_{K / \mathbb{Q}} B_{\gamma}$ is $\mathbb{Q}$-isogenous to the square of a product of two modular abelian varieties with field of Fourier coefficients equal to $\mathbb{Q}(\sqrt{6})$. In $S_{2}\left(\Gamma_{0}\left(2^{4} \cdot 3^{5}\right)\right)$ we find a newform with field of Fourier coefficients $\mathbb{Q}(\sqrt{6})$ and Fourier expansion

$$
g=q+\sqrt{6} q^{5}-2 q^{7}+\sqrt{6} q^{11}-q^{13}+3 \sqrt{6} q^{17}+q^{19}-\sqrt{6} q^{23}+\ldots
$$

Let $\varepsilon$ be the quadratic Dirichlet character of conductor 8 satisfying $\varepsilon(3)=\varepsilon(5)=-1$. Let $h=g \otimes \varepsilon$, which is a newform in $S_{2}\left(\Gamma_{0}\left(2^{6} \cdot 3^{5}\right)\right)$. In the following table we list some local factors 
of the $L$-functions corresponding to the varieties $B_{\gamma} / K, A_{g} / \mathbb{Q}$ and $A_{h} / \mathbb{Q}$.

\begin{tabular}{|l|l|l|l|}
\hline$p$ & $L_{p}\left(B_{\gamma} / K, T\right)^{-1}=L_{p}\left(A_{\gamma} / \mathbb{Q}, T\right)^{-1}$ & $L_{p}\left(A_{g} / \mathbb{Q}, T\right)^{-1}$ & $L_{p}\left(A_{h} / \mathbb{Q}, T\right)^{-1}$ \\
\hline 5 & $\left(1+4 T^{2}+5^{2} T^{4}\right)^{4}$ & $\left(1+4 T^{2}+5^{2} T^{4}\right)$ & $\left(1+4 T^{2}+5^{2} T^{4}\right)$ \\
\hline 7 & $\left(1+2 T+7 T^{2}\right)^{8}$ & $\left(1+2 T+7 T^{2}\right)^{2}$ & $\left(1+2 T+7 T^{2}\right)^{2}$ \\
\hline 11 & $\left(1+16 T^{2}+11^{2} T^{4}\right)^{4}$ & $\left(1+16 T^{2}+11^{2} T^{4}\right)$ & $\left(1+16 T^{2}+11^{2} T^{4}\right)$ \\
\hline 13 & $\left(1-T+13 T^{2}\right) 4\left(1+T+13 T^{2}\right) 4$ & $\left(1+T+13 T^{2}\right)^{2}$ & $\left(1-T+13 T^{2}\right)^{2}$ \\
\hline 17 & $\left(1-20 T^{2}+17^{2} T^{4}\right) 4$ & $\left(1-20 T^{2}+17^{2} T^{4}\right)$ & $\left(1-20 T^{2}+17^{2} T^{4}\right)$ \\
\hline 19 & $\left(1-T+19 T^{2}\right)^{4}\left(1+T+19 T^{2}\right)^{4}$ & $\left(1-T+19 T^{2}\right)^{2}$ & $\left(1+T+19 T^{2}\right)^{2}$ \\
\hline 23 & $\left(1+40 T^{2}+23^{2} T^{4}\right)^{4}$ & $\left(1+40 T^{2}+23^{2} T^{4}\right)$ & $\left(1+40 T^{2}+23^{2} T^{4}\right)$ \\
\hline 29 & $\left(1+34 T^{2}+29^{2} T^{4}\right)^{4}$ & $\left(1+34 T^{2}+29^{2} T^{4}\right)$ & $\left(1+34 T^{2}+29^{2} T^{4}\right)$ \\
\hline 31 & $\left(1-T+31 T^{2}\right)^{8}$ & $\left(1-T+31 T^{2}\right)^{2}$ & $\left(1-T+31 T^{2}\right)^{2}$ \\
\hline 37 & $\left(1-8 T+37 T^{2}\right)^{4}\left(1+8 T+37 T^{2}\right)^{4}$ & $\left(1-8 T+37 T^{2}\right)^{2}$ & $\left(1+8 T+37 T^{2}\right)^{2}$ \\
\hline 41 & $\left(1+58 T^{2}+41^{2} T^{4}\right)^{4}$ & $\left(1+58 T^{2}+41^{2} T^{4}\right)$ & $\left(1+58 T^{2}+41^{2} T^{4}\right)$ \\
\hline
\end{tabular}

We have checked the equality of the local factors of the $L$-functions of $A_{\gamma}$ and $A_{g}^{2} \times A_{h}^{2}$ for all primes $p<1000(p \neq 2,3)$ and this suggests that $A_{\gamma} \sim_{\mathbb{Q}} A_{g}^{2} \times A_{h}^{2}$.

Comparing the local factors $L_{p}(B / K, T)$ and $L_{p}\left(B_{\gamma} / K, T\right)$ we can also find a modular form $f$ such that $A \sim \sim_{\mathbb{Q}} A_{f}^{2}$ as a twist of $g$. More precisely, let $\psi$ be the Dirichlet character of order 4 and conductor 16 such that $\psi(3)=-\sqrt{-1}$ and $\psi(5)=\sqrt{-1}$. The modular form $f=g \otimes \psi$ is a newform in $S_{2}\left(\Gamma_{0}\left(2^{8} \cdot 3^{5}\right), \psi^{2}\right)$ and the local factors $L_{p}(B / K, T)$ and $L_{p}\left(A_{f}, T\right)^{2}$ coincide for all primes $p<1000(p \neq 2,3)$.

A concrete example: $j=-4 / 27$. We now consider another example, corresponding to the stated value of $j$. The Jacobian $B$ of the curve $C_{j}$ is also a building block completely defined over $K=\mathbb{Q}(\sqrt{-6}, \sqrt{-3})$, but a similar analysis shows that in this case the only possibilities for $\left[c_{B / K}\right]_{ \pm}$have $c=1$ in the expression (6.5) , and therefore $\left[c_{B / K}\right]_{ \pm}$is not symmetric. This means that no variety in the isogeny class of $B$ is strongly modular over $K$. If we let for instance $L=K(\sqrt{-1})$ it is easy to see that there exist symmetric elements of $H^{2}(L / \mathbb{Q},\{ \pm 1\})$ whose inflation to $G_{\mathbb{Q}}$ is $\left[c_{B}\right]_{ \pm}$, and then by Proposition 3.4 and Theorem 5.3 there exists a variety isogenous to $B$ completely defined and strongly modular over $L$.

Thus, in this case we have seen that it is enough to go to a quadratic extension $L$ of $K$ to find a variety in the isogeny class of $B$ that is strongly modular over L. However, in the family $\left\{B_{j}\right\}_{j \in \mathbb{Q}}$ we can find varieties where any minimal field $L$ with this property is arbitrarily large. In fact, by Theorem 5.4 this is equivalent to find in this family varieties where the degree of any splitting field is arbitrarily large. We will see this by means of the following lemma.

Lemma 6.5. Let $r \geqslant 2$ be an integer and let $p$ be a prime such that $p \equiv 1\left(\bmod 2^{r}\right)$ and $p \equiv-1$ $(\bmod 3)$. Then the order of any splitting character for $B_{1 / p}$ is at least $2^{r}$.

Proof. For simplicity we call $B$ the variety $B_{1 / p}$, and let $\left[c_{B}\right]$ be its attached cohomology class. By (6.4) the sign component $\left[c_{B}\right]_{ \pm}$is given as the following product of quaternion algebras:

$$
\left[c_{B}\right]_{ \pm}=(-(27+16 p) / p, 3)_{\mathbb{Q}} \cdot(-(27+16 p), 2)_{\mathbb{Q}} \cdot(2,3)_{\mathbb{Q}} \cdot
$$

Applying the formulas for computing the local Hilbert Symbols at $p$ we find that

$$
(-(27+16 p) / p, 3)_{p}=-1, \quad(-(27+16 p), 2)_{p}=1, \quad(2,3)_{p}=1,
$$

and this implies that the local component of $\left[c_{B}\right]_{ \pm}$at the prime $p$ is -1 . But $\left[c_{B}\right]_{ \pm}=\left[c_{\varepsilon}\right]$, where $\varepsilon$ is the splitting character associated to any splitting map $\alpha$ for $B$. We can identify $\varepsilon$ with a primitive Dirichlet character of a certain conductor $N$, and if $\varepsilon_{p}$ denotes the component of 
$\varepsilon$ modulo the largest power of $p$ dividing $N$, then the local component of $\left[c_{\varepsilon}\right]$ at $p$ is given by $\varepsilon_{p}(-1)$. The value $\varepsilon_{p}(-1)=-1$ is taken by the characters of order multiple of $2^{\operatorname{ord}_{2}(p-1)}$, and it follows that $\operatorname{ord}(\varepsilon) \geqslant \operatorname{ord}\left(\varepsilon_{p}\right) \geqslant 2^{\operatorname{ord}_{2}(p-1)}$, which is at least $2^{r}$ by our choice of $p$.

Proposition 6.6. For any integer $r$ there exists a variety $B$ in the family $\left\{B_{j}\right\}_{j \in \mathbb{Q}}$ such that any splitting field for $B$ has degree at least $2^{r}$.

Proof. Take a prime $p$ as in the previous lemma, and take as $B$ the variety $B_{1 / p}$. Let $\alpha$ be any splitting map for $B$, and let $\varepsilon$ be its associated splitting character. Then we have that $\left[K_{\alpha}: \mathbb{Q}\right] \geqslant\left[K_{\varepsilon}: \mathbb{Q}\right] \geqslant 2^{r}$.

From Lemma 6.5 we can derive another interesting consequence.

Proposition 6.7. Let $g$ be any natural number. There exist varieties $B$ in the family $\left\{B_{j}\right\}_{j \in \mathbb{Q}}$ such that every $\mathbb{Q}$-simple abelian variety $A$ of $\mathrm{GL}_{2}$-type having $B$ as its simple factor is of dimension $\operatorname{dim} A \geqslant g$.

Proof. Let $r$ be an integer such that $\varphi\left(2^{r}\right)=2^{r-1} \geqslant g$, and take $B=B_{1 / p}$ with $p$ a prime as in Lemma 6.5. If $A$ is a simple abelian variety of $\mathrm{GL}_{2}$-type that has $B$ as its simple factor, the field $E=\operatorname{End}_{\mathbb{Q}}^{0}(A)$ is isomorphic to $E_{\alpha}$ for some splitting map $\alpha$ for $B$. The field $E_{\alpha}$ contains the values of the splitting character $\varepsilon$ associated to $\alpha$. Therefore, it contains the $2^{r}$-th cyclotomic extension and we have that $\operatorname{dim} A=\left[E_{\alpha}: \mathbb{Q}\right] \geqslant \varphi\left(2^{r}\right) \geqslant g$.

\section{REFERENCES}

[1] S. Baba, H. Granath. Genus 2 curves with quaternionic multiplication. Canad. J. Math. 60 (2008), no. 4, pp. 734-757.

[2] C. Breuil, B. Conrad, F. Diamond, R. Taylor. On the modularity of elliptic curves over $\mathbb{Q}$ : wild 3-adic exercises. J. Amer. Math. Soc. 14 (2001), no. 4, 843-939

[3] J. Cremona. Algorithms for modular elliptic curves. Second edition, Cambridge University Press (1997). Available online at www.warwick.ac.uk/ masgaj/book/fulltext/index.html.

[4] L. Dieulefait, J. Jiménez-Urroz. Solving Fermat-type equations via modular $\mathbb{Q}$-curves over polyquadratic fields. J. Reine Angew. Math. 633 (2009), pp. 183-195.

[5] N. Elkies. On elliptic K-curves. Modular curves and abelian varieties, Progress in Math., vol. 224 (2002), pp. 81-91.

[6] J. González, J.-C. Lario. Rational and elliptic parametrizations of $\mathbb{Q}$-curves. J. Number Theory 72 (1998), no. 1 , pp. 13-31.

[7] J. González, J.-C. Lario. $\mathbb{Q}$-curves and the Manin ideal. Amer. J. Math. 123 (2001), no. 3, pp. 475-503.

[8] J. González, J.-C. Lario. Modular elliptic directions with complex multiplication (with an application to Gross's elliptic curves). Comment. Math. Helv. 86 (2011), no. 2, 317-351.

[9] K.-I. Hashimoto, N. Murabayashi. Shimura curves as intersections of Humbert surfaces and defining equations of QM-curves of genus two. Tôhoku Math. J. 47 (1995), no. 2, pp. 272-295.

[10] G. Karpilovsky. Group representations, vol. 2. North-Holland Mathematics Studies, 177. North-Holland Publishing Co., Amsterdam, 1993.

[11] C. Khare, J.-P. Wintenberger. Serre's modularity conjecture. I. Invent. Math. 178 (2009), no. 3, pp. $485-504$.

[12] C. Khare, J.-P. Wintenberger. Serre's modularity conjecture. II. Invent. Math. 178 (2009), no. 3, pp. 505-586.

[13] J. S. Milne. Complex Multiplication. Course notes available at www.jmilne.org.

[14] D. Mumford. Abelian varieties. Second edition, Oxford University Press, London, 1974.

$[15]$ E. E. Pyle. Abelian varieties over $\mathbb{Q}$ with large endomorphism algebras and their simple components over $\overline{\mathbb{Q}}$. Modular curves and abelian varieties, Progress in Math., vol. 224 (2002), pp. 189-239.

[16] J. Quer. $\mathbb{Q}$-curves and abelian varieties of $\mathrm{GL}_{2}$-type. Proc. London Math. Soc. 81 (2000) no. 2, pp. $285-317$.

[17] J. Quer. Embedding problems over abelian groups and an application to elliptic curves. J. Algebra 237 (2001), no. 1, pp. 186-202.

[18] J. Quer. Fields of definition of building blocks. Math. Comp. 78 (2009), pp. 537-554.

[19] K. Ribet, Fields of definition of abelian varieties with real multiplication. Arithmetic geometry (Tempe, AZ, 1993), pp. 107-118, Contemp. Math., 174, Amer. Math. Soc., Providence, RI, 1994. 
[20] K. A. Ribet. Abelian varieties over $\mathbb{Q}$ and modular forms. Modular curves and abelian varieties, Progress in Math., vol. 224 (2002), pp. 241-261.

[21] G. Shimura. Introduction to the arithmetic theory of automorphic functions. Publ. Math. Soc. Japan, vol. 11, Princeton University Press (1971).

[22] W. Stein. Modular forms: A computational approach, with an appendix by Paul Gunnells. AMS Graduate Studies in Mathematics, vol. 79. (2007).

[23] R. Taylor, A. Wiles. Ring-theoretic properties of certain Hecke algebras. Ann. of Math. 141 (1995), no. 3, pp. 553-572.

[24] A. Wiles. Modular elliptic curves and Fermat's last theorem. Ann. of Math. 141 (1995), no. 3, pp. 443-551.

X. G.: Max Planck Institute for Mathematics, Vivatsgasse 7, 53111 Bonn, Germany and Departament de Matemàtica Aplicada II, Universitat Politècnica de Catalunya, C. Jordi Girona 1-3, 08034 Barcelona, Spain

E-mail address: xevi.guitart@gmail.com

J. Q.: Departament de Matemàtica Aplicada II, Universitat Politècnica de Catalunya, C. Jordi Girona 1-3, 08034 BARCELONA, Spain

E-mail address: jordi.quer@upc.edu 